\title{
Confinement of FRP concrete columns: Review of design guidelines and comparison with experimental results
}

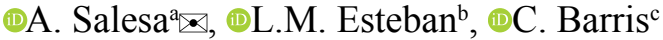 \\ a. Dept. of Materials and Structures, Engineering School of La Almunia, Universidad de Zaragoza, (Zaragoza, Spain) \\ b. Dept. of Applied Mathematics, Engineering School of La Almunia, Universidad de Zaragoza, (Zaragoza, Spain) \\ c. Dept. of Mechanical Engineering and Industrial Construction, University of Girona (UdG), (Girona, Spain) \\ ه: asalebor@yahoo.es
}

\begin{abstract}
A regulatory framework is required to ensure the correct design of Fibre-Reinforced Polymers (FRPs) increasingly being used as an externally-bonded strengthening system on concrete columns. Several design guidelines on the confinement of FRP concrete have been developed over the past few years worldwide, each proposing a different approach, resulting in different predictions. This study aims to evaluate and compare nine international design guidelines used to predict the compressive strength of confined concrete in FRP-strengthened concrete columns and weigh them against experimental results. The results of this investigation reveal that the predictions from the guidelines on the compressive strengthening of FRP-confined concrete are generally suitable for circular columns, with the ACI-440 and CNR-DT 200 guideline predictions being two of the most accurate. Nevertheless, the guidelines generally tend to overestimate the load-carrying capacity for the compressive strength of FRP-confined concrete in non-circular columns, for which further experimental work using large-scale specimens is required.
\end{abstract}

KEYWORDS: Concrete; Composite; FRP; Confinement; Compressive Strength.

Citation/Citar como: Salesa, A.; Esteban, L.M.; Barris, C. (2022) Confinement of FRP concrete columns: Review of design guidelines and comparison with experimental results. Mater. Construcc. 72 [345], e274. https://doi.org/10.3989/mc.2022.03821.

RESUMEN: Confinamiento de columnas de hormigón con FRP: revisión de normativas y sus predicciones. El crecimiento en el uso de polímeros reforzados con fibra (FRP) como sistema de refuerzo externo en columnas de hormigón requiere de un marco regulatorio para su correcto diseño. En los últimos años se han desarrollado diferentes guías y normativas de diseño, teniendo cada una de ellas un planteamiento diferente y, por lo tanto, arrojando resultados dispares. Esta investigación pretende contrastar con resultados experimentales las predicciones que nueve normativas internacionales hacen sobre la resistencia a compresión de una columna de hormigón confinada con FRP. Los resultados de la investigación muestran que las estimaciones de las normativas sobre la resistencia de las columnas de hormigón confinadas con FRP son, en general, adecuadas para columnas circulares; destacan como las más precisas la ACI-440 y la CNR-DT 200. Por contra, en el caso de columnas no circulares los resultados obtenidos tienden a sobreestimar la resistencia a compresión de las columnas confinadas con FRP, en este caso sería necesario continuar investigando en modelos a gran escala.

PALABRAS CLAVE: Hormigón; Composite; FRP; Confinamiento; Resistencia a la compresión.

Copyright: (C2022 CSIC. This is an open-access article distributed under the terms of the Creative Commons Attribution 4.0 International (CC BY 4.0) License. 


\section{INTRODUCTION}

One of the main causes of buildings collapsing is column failure under concentric axial load or due to small eccentricities (1). Rehabilitation and strengthening systems have been developed and used to prevent structural failure on existing deteriorated concrete structures or to increase their design load. The column strengthening and retrofitting process has traditionally used two methods: use of external steel angles and horizontal strips or addition of a new concrete jacket with additional reinforcement $(2,3)$ stress levels were achieved that produced intemal microcracks, which allowed residual rigidity and the behaviour of completely microcraked concrete specimens to be studied. The specimens were subsequently tested to compression to the fracture point. Specimens reinforced in accordance with no manufacturing defects (100\% CFRP reinforcement). Both methods are expensive and time-consuming as they are labour intensive (4). Moreover, both solutions have drawbacks because the useful floor area is reduced and steel strengthening requires protection against corrosion.

When subject to a compressive axial load, a concrete column tends to expand laterally due to the Poisson effect. When a column is FRP-strengthened, this lateral expansion is limited by the FRP due to the development of lateral confinement stress (passive confinement) (5). An FRP-confined concrete column behaves in a state of triaxial compressive stress, increasing hydrostatic stress and reducing deviatoric stress. Consequently, the development of internal microcracks is limited (6), which improves compressive strength and ductility, resulting in better structural capacity of the column. Owing to all the potential advantages of FRP, extensive experimental and analytical work has been carried out over the past 30 years (7). Based on conclusions from the research, major efforts have been made to develop design guidelines and specifications for FRP-wrapped concrete columns. The design guidelines provide various equations that give varying results, some of which differ significantly from the experimental results. Therefore, further research and guideline validation is required to improve construction industry confidence in technology that uses FRP strengthening (8).

This study analyses the most up-to-date international design guidelines for concrete columns fully-wrapped with FRP under concentric axial loads. The goals of this research are to: (i) provide an indepth description of the guidelines, (ii) compare the predictions of the design guidelines against each other and against the experimental test results from previous research and (iii) identify and highlight the strengths and weaknesses of the design guidelines. For this purpose, a theoretical background is firstly presented. Secondly, the design guidelines are reviewed. Thirdly, a comparative study of design guideline estimations is provided. Lastly, conclusions and recommendations on design guideline use are given.

\section{THEORETICAL BACKGROUND}

Studying the beneficial effect of lateral confinement on the strength and deformation capacity of concrete dates back to the early $20^{\text {th }}$ century. In 1901 , the engineer Considère studied the concept of béton fretté [concrete confinement in encased reinforced concrete columns] (9-12). Some years later, Richart et al. carried out an extensive study about lateral active confinement on concrete cylinders under simple compression (13). They determined that both compressive strength and strain increments are directly related to the lateral confinement pressure applied (Equations [1] and [2], respectively).

$$
\begin{gathered}
\frac{f_{c c}^{\prime}}{f_{c o}^{\prime}}=1+k_{1} \frac{f_{l}}{f_{c o}^{\prime}} \text { with } k_{1}=4.1 \\
\frac{\varepsilon_{c c u}}{\varepsilon_{c}^{\prime}}=1+k_{2}\left(\frac{f_{c c}^{\prime}}{f_{c o}^{\prime}}-1\right) \text { with } k_{2}=5[2]
\end{gathered}
$$

In Equations [1] and [2] above, $f_{c c}^{\prime}$ is the compressive strength of confined concrete, $f_{c o}^{\prime}$ is the compressive strength of unconfined concrete, $f_{l}$ is the confinement pressure, $\varepsilon_{c}^{\prime}$ is the comprresive strain of unconfined concrete, $\varepsilon_{c c u}$ is the ultimate axial compressive strain of confined concrete and $k_{1}$ and $k_{2}$ are efficiency factors.

In 1988, Mander et al. (14) tested circular, square and rectangular reinforced concrete columns and developed a model that predicted confined concrete strength values (Equation [3]).

$$
\frac{f_{c c}^{\prime}}{f_{c o}^{\prime}}=2.254 \sqrt{1+7.94 \frac{f_{l}}{f_{c o}^{\prime}}}-2 \frac{f_{l}}{f_{c o}^{\prime}}-1.254 \text { [3] }
$$

Generally, the column-strengthening effect can be classed as active or passive confinement. Active confinement involves applying external confinement pressure. Conversely, passive confinement is a result of the Poisson effect that takes place when the column starts to dilate and transverse deformation is totally or partially limited (15).

The FRP confinement mechanism is classed as passive confinement derived from an equilibrium of forces. In an FRP-confined circular concrete section with the FRP shell fibre perpendicular to the column axis, lateral confinement pressure is assumed to be uniformly distributed around the circumference. Lat- 
eral pressure starts to be applied to the FRP wrap due to the lateral expansion of concrete under an axial compression load. Therefore, the FRP confinement action implemented is of the passive type. Confinement pressure grows in proportion to lateral expansion, up to FRP shell failure. Theoretically, the maximum confinement pressure is deduced from a force equilibrium (Equation [4]), which is directly related to the FRP's modulus of elasticity $\left(E_{f}\right)$, ply thickness $\left(t_{f}\right)$, number of plies $(n)$ and effective FRP strain or hoop rupture strain $\left(\varepsilon_{f e}\right)$ and inversely related to the circular section diameter $(D)$.

$$
f_{l}=\frac{2 E_{f} n t_{f} \varepsilon_{f e}}{D}
$$

It is important to point out that effective FRP strain is substantially lower than the ultimate tensile strain of the fibres $\left(\varepsilon_{f u}\right)$ measured using a flat FRP coupon tensile test (16). The relationship between both is generally established via a strain reduction or efficiency factor $\left(k_{\varepsilon}\right)$, as shown in Equation [5].

$$
\varepsilon_{f e}=k_{\varepsilon} \varepsilon_{f u}
$$

According to Lam and Teng $(17,18)$, the difference between the two strains lies in several factors: i) the effect of FRP jacket curvature, ii) a non-uniform deformation of cracked concrete leading to non-uniform stress distribution in the FRP jacket and its subsequent premature rupture and iii) the existence of an overlapping zone where the strain measured is much lower than the strain measured in other FRP sheet zones. fib Bulletin No. 14 (19) also states that ultimate tensile strain reduction of FRP may be influenced by the quality of implementation (fibre incorrectly aligned and damage to fibre due to edges or local protrusions) and the size effect (when multiple layers are applied). In research by Realfonzo and Napoli (20), the same average strain efficiency factor value $\left(k_{\varepsilon}=0.60\right)$ for carbon and glass FRP jackets was obtained, regardless of concrete strength. Further to analysing several stress-strain models for FRP-confined concrete in circular sections, Ozbakkaloglu et al. (21) determined that the average strain efficiency factor was between 0.641 and 0.685 .

Due to the characteristics of passive FRP confinement, a strength increment for unconfined concrete is not always provided. As such, the minimum confinement ratio, the relationship between confinement pressure and unconfined concrete strength, is established.

According to Lam and Teng (18), when a specimen is insufficiently confined in the stress-strain curve, there is a branch that descends after the peak, which requires the confined concrete stress at ultimate strain $\left(f_{c u}^{\prime}\right)$ to be smaller than both the unconfined concrete strength $\left(f_{c o}^{\prime}\right)$ and the maximum confined concrete strength $\left(f_{c c}^{\prime}\right)$ (Figure 1, line c). Alternatively, if $f_{c u}^{\prime}$ is larger than $f_{c o}^{\prime}$, the concrete is sufficiently confined and two stress-strain curves are possible: a) a curve with two ascending branches where: $f_{c u}^{\prime}=f_{c c}^{\prime}$ (Figure 1 , line a) and b) a curve with an initial ascending branch and a second descending branch. For the second case: $f_{c o}^{\prime}<f_{c u}^{\prime}<f_{c c}^{\prime}$ (Figure 1, line b).

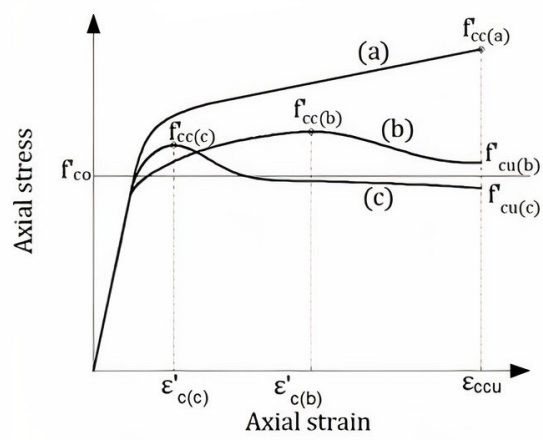

FIGURE 1. Schematic stress-strain behaviour of (a) sufficiently confined concrete with $\mathrm{f}_{\mathrm{cu}}^{\prime}=\mathrm{f}_{\mathrm{cc}}^{\prime}$; (b) sufficiently confined concrete with $\mathrm{f}_{\mathrm{co}}^{\prime}<\mathrm{f}_{\mathrm{cu}}^{\prime}<\mathrm{f}_{\mathrm{cc}}^{\prime}$; (c) insufficiently confined concrete with $\mathrm{f}_{\mathrm{cu}}^{\prime}<\mathrm{f}_{\mathrm{co}}^{\prime}$. (adapted from Lam and Teng (18)).

For this reason, a minimum confinement ratio is proposed in the FRP confinement models and design guidelines. The strength gain for confined concrete with respect to unconfined concrete is measured via the strength enhancement ratio $\left(f_{c c}^{\prime} / f_{c o}^{\prime}\right)$. The final confined strength depends on lateral FRP pressure and unconfined concrete strength, as shown in Equation [1]. The improvement observed in the maximum confined concrete strength of FRP-wrapped columns decreases as unconfined concrete strength increases (22-25).

Several stress-strain models have been presented for FRP-confined concrete. According to Lam and Teng (18), most of these models can be classified into two groups: design-oriented and analysis-oriented. Design-Oriented Models (DOMs) are based on interpreting the experimental results directly. Through regression analysis and test calibrations, they propose closed-form equations for predicting confined concrete strength, ultimate strain and the stress-strain response. Analysis-Oriented Models (AOMs) are active confinement models used to evaluate passively-confined concrete using FRP. They are based on a force equilibrium and radial displacement compatibility requiring an incremental numerical procedure. According to Teng et al. (26), AOMs are more versatile and powerful than DOMs as they explicitly consider the response of the concrete and the FRP jacket as well as their interactions. Moreover, they can predict the behaviour of sufficiently and insufficiently confined concrete. However, the complexity of the incremental process discourages their direct use in design calculations (18). 
Jiang and Teng (27) conducted a detailed review of the eight most common AOMs, stating that the AOM model of Teng et al. (26) had the best performance. In turn, Ozbakkaloglu et al.(21) assessed 68 stress-strain models for FRP-confined concrete in circular sections, stating that DOMs perform better than AOMs. They also concluded that the DOM models presented by Lam and Teng (18) and Tamuzs et al. (28) were the most accurate for predicting the ultimate strength and strain enhancement ratios, respectively.

Most experimental and theoretical studies in the literature focus on FRP concrete confinement for circular sections $(29,30)$ However, it is also common to design columns with a rectangular or square cross-section (31). It is well accepted that FRP confinement is less effective for rectangular columns than for circular columns $(16,32,33)$; in circular sections, the FRP jacket uniformly confines the concrete whilst in rectangular sections it is non-uniformly confined as FRP confinement is mainly concentrated at the column corners. Only some of the concrete column core is effectively confined and, as a result, confinement effectiveness is reduced (34). It is generally accepted that the effective confinement area is contained within four second-degree parabolas (Figure 2). Outside these parabolas, negligible confinement occurs (30).

FRP-confined stress-strain models, compressive strength and ultimate strain equations for rectangular sections are usually based on the definition of an equivalent circular column. They introduce an equivalent diameter $\left(D_{\text {eqv }}\right)$ and a shape factor $\left(k_{s}\right)$ to take account of the effect of non-uniform confinement (30, $34,35)$. In confined rectangular cross-section areas, FRP rupture generally occurs at the corners or close to them due to a concentration of stress in these areas (36). Confinement effectiveness improves with larger corner radii $\left(r_{c}\right)(25,37)$ and with a smaller dimensional aspect ratio (ratio between longer and shorter sides $(h / b))(16,38)$.

It is important to emphasise that previous comments are based on observations mainly made on small-scale cylindrical test specimens. Few studies have worked on a large or medium scale (39) due to high costs and a lack of high-capacity testing equipment (36). For circular columns, column size has no significant effect on observed behaviour according to a limited number of existing tests on large-scale columns. However, for rectangular columns, the effect of column size is uncertain due to the very limited data $(16,40)$.

\section{DESIGN GUIDELINE REVIEW}

The following design guidelines are reviewed in this document: ACI-440.2R-17 (41), AFGC-11 (42), CNR-DT-200-R1-13 (43), CS-TR55-12 (44), CSA-S806-12 (45), fib-B90-19 (46), ISIS-DM4-08 (47), NCHRP-R655-10 (48) and TEC-07 (49).

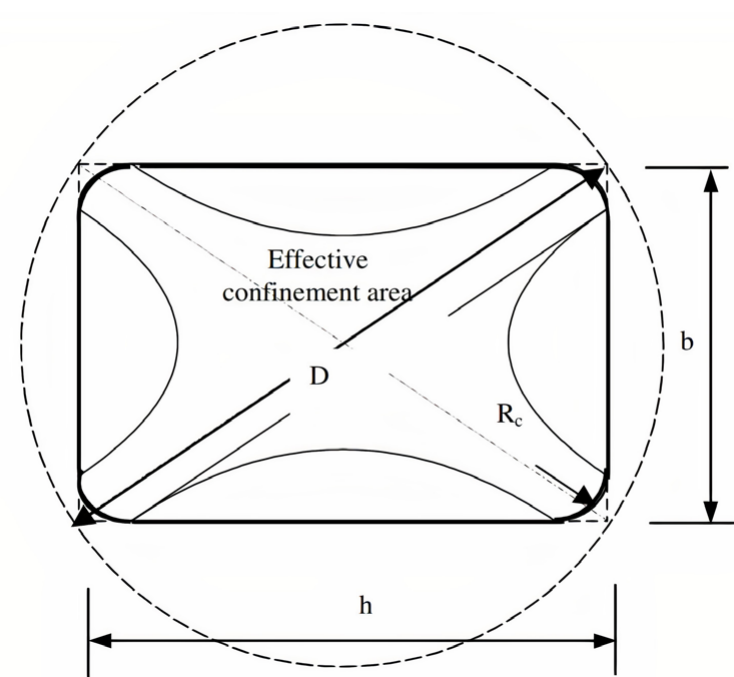

FIGURE 2. Effective confinement area and equivalent diameter of rectangular cross section by Lam and Teng (30).

In the presentation and discussion of the design methods provided by the aforementioned guidelines, the notation and definitions have been made uniform for consistency and simplicity. The new harmonized parameters may differ from the originals, but the results are not affected in any way.

This work mainly focuses on studying the increase in compressive concrete column strength due to FRP confinement. The ultimate or design axial column capacity is not considered in this study, meaning that both the partial and global safety factors (based on the limit state design philosophy) are outside the scope of this research. Only the reduction factors related to computing lateral confinement pressure and, as a result, confined concrete strength, are considered. These additional FRP factors and other safety factors can be found in Table 1 for information purposes only. The subscripts "c", "s" and "f" indicate concrete, steel and FRP, respectively.

The model types and limitations used in each design guideline are presented in Table 2. The limitations for each cross-sectional type relate to the compressive concentric axial load, including the maximum side column dimensions, the maximum side-aspect ratio $(h / b)$ and the minimum corner radius of the prismatic cross-section. All the guidelines give a recommendation on the maximum aspect ratio between large the long and short sides. Regarding the corner radius, all guidelines except for the TEC guideline give a minimum value (from $13 \mathrm{~mm}$ in the ACI guideline to 35 $\mathrm{mm}$ in the AFGC and ISIS guidelines).

A summary of the expressions for concrete column FRP confinement is given in Tables 3 and 4 for circular and non-circular cross-sections, respectively. The tables include all the instructions that the guidelines provide for effective confinement pressure $\left(f_{l}\right)$ , maximum compressive strength $\left(f_{c c}^{\prime}\right)$ and ultimate axial strain $\left(\varepsilon_{c c u}\right)$ for FRP-confined reinforced concrete columns. 
TABLE 1. Strength reduction and material safety factors for different guidelines.

\begin{tabular}{|c|c|c|c|}
\hline Guideline & Strength reduction factors & Materials safety factors & FRP additional factors \\
\hline ACI -17 & $\begin{array}{c}\varphi=0.75 \\
\text { (spiral transverse reinfor. type (52)) } \\
\varphi=0.65 \\
\text { (other transverse reinfor. type (52)) }\end{array}$ & NA & $\begin{array}{c}\Psi_{\mathrm{f}}=0.95 \\
\mathrm{C}_{\mathrm{E}}=\text { environmental factor (function of } \\
\text { the exposure conditions and material: } \\
0.95-0.50 \text { ) } \\
f_{f u}=C_{e} f_{f u}^{*} / \varepsilon_{f u}=C_{e} \varepsilon_{f u}^{*} / E_{f}=f_{f u} / \varepsilon_{f u}\end{array}$ \\
\hline AFGC - 11 & NA & $\begin{array}{l}\text { Safety coefficients depend on FRP } \\
\text { type of limit state combination } \\
\qquad \begin{array}{c}\gamma_{c}=1.5 / 1.2 \\
\gamma_{s}=1.15 / 1.0 \\
\gamma_{f}=\text { from } 1.0 \text { to } 2.5\end{array}\end{array}$ & Ageing factor: $\alpha_{f}=0.65$ \\
\hline CNR - 13 & NA & $\begin{array}{l}\gamma_{c}=1.5 \\
\gamma_{s}=1.15 \\
\gamma_{f}=1.10\end{array}$ & $\begin{array}{l}\eta_{a} \text { : Environmental factor dependent of } \\
\text { type of fiber/resin and exposure condi- } \\
\text { tions. From } 0.50 \text { to } 0.95 \text {. }\end{array}$ \\
\hline CS -12 & NA & $\begin{array}{c}\gamma_{c}=1.5 \\
\gamma_{s}=1.15 \\
\gamma_{f}=1.1 \text { Carbon and Aramid, } 1.6 \text { AR glass, } \\
1.8 \mathrm{E}-\text { glass and Basalt }\end{array}$ & $\begin{array}{l}\text { There is an additional partial safety fac- } \\
\text { tor, } \gamma_{\mathrm{FRP}, \mathrm{m}} \text {, to be applied to manufactured } \\
\text { composites. There is also a partial safety } \\
\text { factor for ultimate strain, } \gamma_{\mathrm{FRP}, \varepsilon} \\
\qquad \varepsilon_{f u}=\frac{\varepsilon_{f k}}{\gamma_{F R, \varepsilon} \gamma_{F R P, m}}\end{array}$ \\
\hline CSA - 12 & NA & $\begin{array}{l}\phi_{c}=0.65 \\
\phi_{s}=0.85 \\
\phi_{f}=0.75\end{array}$ & $\varphi_{\mathrm{f}}=0.75 \mathrm{FRP}$ resistance factor \\
\hline fib - 19 & NA & $\gamma_{f}=1.25$ & NA \\
\hline ISIS - 08 & NA & $\begin{array}{c}\phi_{c}=0.75 \\
\phi_{s}=0.95 \\
\phi_{f}=\text { depends on material } \\
\text { and installation process } \\
(\text { CFRP }=0.56 / \text { AFRP }=0.38 / \text { GFRP }=0.49)\end{array}$ & $\varphi_{\mathrm{f}}=$ embedded in the $f_{l}$ limit equation \\
\hline $\begin{array}{l}\text { NCHRP } \\
-10\end{array}$ & $\begin{array}{l}\varphi \text { according to AASHTO LRFD } \\
\text { Bridge Design Specification (53) }\end{array}$ & NA & $\begin{array}{c}\varphi_{\mathrm{f}}=0.65 \mathrm{FRP} \text { resistance factor embedded } \\
\text { in the } f_{l} \text { equation }\end{array}$ \\
\hline TEC-07 & $\begin{array}{r}\text { Safety Factors in } \\
\text { TS-498 / TS-500 / TS-70 }\end{array}$ & $\begin{array}{l}\text { Turkish Standards: } \\
\text { / TS - } 3233 \text { and TS-9967 }\end{array}$ & NA \\
\hline
\end{tabular}

Depending on the guideline, the value to be used for unconfined concrete strength $\left(f_{c o}^{\prime}\right)$ has been considered to be the characteristic concrete compressive strength determined based on a standard cylinder $\left(f_{c}^{\prime}\right)$ or $85 \%$ of $f_{c}^{\prime}$. The CSA and CS guideline expressions use the coefficient 0.85 , whilst the other guidelines use $f_{c}^{\prime}$. The ACI guideline specifies that $f_{c o}^{\prime}$ is equal to $0.85 f_{c}^{\prime}$. However, the given expressions for FRP confinement are based on $f_{c}^{\prime}$ without the 0.85 factor.

With respect to this, Légeron and Paultre (50) pointed out that there is a difference between the strength of in-situ concrete and the strength determined based on the standard cylinder test; for columns under axial compression, a coefficient of 0.85 is the commonly-accepted value (51).

\section{COMPARATIVE STUDY}

In this section, a comparative study is carried out to assess the suitability of the analysed guidelines. The comparative study is conducted in two stages: i) a theoretical parametric study of the guideline expressions and ii) an experimental comparative study of the guideline estimations and experimental results. In the subsequent comparative sections, the following apply:

- No safety factors, for both materials and forces, are applied when calculating FRP concrete confinement.

- No environmental or ageing factors are considered in the calculations. 
TABLE 2. Design guidelines limitations and type of models.

\begin{tabular}{|c|c|c|c|c|c|}
\hline Guideline & $\begin{array}{l}\text { Cross-sectional } \\
\text { type }\end{array}$ & Limit & ations & Type of model & \\
\hline \multirow[b]{2}{*}{ ACI -17} & Circular & \multirow{2}{*}{$\begin{array}{l}\text { Concentric axial load } \\
\text { Fully wrapped } \\
f_{c}^{\prime} \leq 70 \mathrm{MPa}\end{array}$} & None & $\begin{array}{l}\text { Stress-strain, strength enhancement } \\
\text { and maximum strain }\end{array}$ & \multirow{2}{*}{$\begin{array}{c}\text { Lam and Teng } \\
(18),(30) \\
\text { Spoelstra and } \\
\text { Monti (54) }\end{array}$} \\
\hline & Prismatic & & $\begin{array}{c}h / b \leq 2.0 \\
(h, b) \leq 900 \mathrm{~mm} \\
r_{c, \min } \geq 13 \mathrm{~mm}\end{array}$ & $\begin{array}{l}\text { Strength enhancement and max- } \\
\text { imum strain }\end{array}$ & \\
\hline \multirow[b]{2}{*}{ AFGC - 11} & Circular & \multirow{2}{*}{$\begin{array}{l}\text { Concentric axial load } \\
\text { Fully or partially } \\
\text { wrapped }\end{array}$} & None & Strength enhancement & \multirow[b]{2}{*}{ Not specified } \\
\hline & Prismatic & & $\begin{array}{c}h / b \leq 1.5 \\
r_{c, \text { min }} \geq 35 \mathrm{~mm}\end{array}$ & Strength enhancement & \\
\hline \multirow[b]{2}{*}{ CNR- 13} & Circular & \multirow{2}{*}{$\begin{array}{l}\text { Concentric axial load } \\
\text { Fully or partially } \\
\text { wrapped }\end{array}$} & None & $\begin{array}{l}\text { Stress-strain, strength enhancement } \\
\text { (maximum strain for axial+bending) }\end{array}$ & \multirow[b]{2}{*}{ Not specified } \\
\hline & Prismatic & & $\begin{array}{c}\mathrm{h} / \mathrm{b} \leq 2.0 \\
(h, b) \leq 900 \mathrm{~mm} \\
r_{c, \min } \geq 20 \mathrm{~mm}\end{array}$ & $\begin{array}{l}\text { Strength enhancement } \\
\text { (maximum strain for axial+bending) }\end{array}$ & \\
\hline \multirow{3}{*}{ CS - 12} & Circular & \multirow{3}{*}{$\begin{array}{l}\text { Concentric axial load } \\
\text { Fully wrapped }\end{array}$} & None & $\begin{array}{l}\text { Stress-strain, strength enhance- } \\
\text { ment and maximum strain }\end{array}$ & \multirow{2}{*}{$\begin{array}{l}\text { Teng et al. (55) } \\
\text { Lam and Teng } \\
\text { (18), (30) }\end{array}$} \\
\hline & Prismatic & & $\begin{array}{c}h / b \leq 1.5 \\
r_{c, \min } \geq 20 \mathrm{~mm}\end{array}$ & Stren atb onbront & \\
\hline & Elliptical & & $a / c \leq 2.5$ & 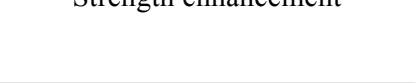 & $\begin{array}{l}\text { Tem and Lang } \\
\text { (56) }\end{array}$ \\
\hline \multirow[b]{2}{*}{ CSA - 12} & Circular & \multirow{2}{*}{$\begin{array}{l}\text { Concentric axial load } \\
\text { Fully wrapped } \\
f_{c}^{\prime} \leq 80 \mathrm{MPa}\end{array}$} & None & & \multirow[b]{2}{*}{ Not specified } \\
\hline & Prismatic & & $\begin{array}{c}f_{c}^{\prime} \leq 80 \mathrm{MPa} \\
h / b \leq 1.5 \\
r_{c, \text { min }} \geq 20 \mathrm{~mm}\end{array}$ & Strength enhancement & \\
\hline \multirow[b]{2}{*}{ fib - 19} & Circular & \multirow{2}{*}{$\begin{array}{l}\text { Concentric axial load } \\
\text { Fully or partially } \\
\text { wrapped }\end{array}$} & $h / b \leq 2.0$ & $\begin{array}{l}\text { Stress-strain, strength and maxi- } \\
\text { mum strain }\end{array}$ & \multirow{2}{*}{$\begin{array}{c}\text { Lam and Teng } \\
(18),(30) \\
\text { Triantafillou et } \\
\text { al. (57) }\end{array}$} \\
\hline & Prismatic & & $\begin{array}{c}r_{c} \text { maximum possible } \\
r_{c, \text { min } ; \text { aramid }} \geq 10 \mathrm{~mm} \\
r_{c, \text { min; } \text { glass or carbon }} \geq 20 \mathrm{~mm}\end{array}$ & Strength and maximum strain & \\
\hline \multirow[b]{2}{*}{ ISIS - 08} & Circular & \multirow{2}{*}{$\begin{array}{l}\text { Concentric axial load } \\
\text { Fully wrapped }\end{array}$} & None & Strength enhancement & \multirow[b]{2}{*}{ Not specified } \\
\hline & Prismatic & & $\begin{array}{c}h / b \leq 1.5 \\
h \leq 800 \mathrm{~mm} \\
r_{c, \min } \geq 35 \mathrm{~mm}\end{array}$ & Strength enhancement & \\
\hline \multirow[b]{2}{*}{ NCHRP - 10} & Circular & \multirow{2}{*}{$\begin{array}{l}\text { Concentric axial load } \\
\text { Fully wrapped }\end{array}$} & $L / D \leq 8$ & \multirow[b]{2}{*}{ Strength enhancement } & \multirow[b]{2}{*}{ Not specified } \\
\hline & Prismatic & & $\begin{array}{c}h / b \leq 1.1 \\
r_{c, \min } \geq 25.4 \mathrm{~mm}\end{array}$ & & \\
\hline \multirow{3}{*}{ TEC - 2007} & Circular & \multirow{3}{*}{$\begin{array}{l}\text { Concentric axial load } \\
\text { Fully wrapped }\end{array}$} & None & \multirow{3}{*}{$\begin{array}{l}\text { Strength enhancement and max- } \\
\text { imum strain }\end{array}$} & \multirow{3}{*}{ Not specified } \\
\hline & Prismatic & & $h / b \leq 2.0$ & & \\
\hline & Elliptical & & $a / c \leq 3.0$ & & \\
\hline
\end{tabular}


Confinement of FRP concrete columns: Review of design guidelines and comparison with experimental results $\bullet$

TABLE 3. Summary of design guidelines' equations for circular cross sections.

\begin{tabular}{|c|c|c|c|}
\hline Guideline & $\begin{array}{c}\text { Effective confinement pressure } \\
f_{l}(\mathrm{MPa})\end{array}$ & $\begin{array}{l}\text { Confined concrete compressive strength } \\
\text { for purpose of design } f_{c c}^{\prime}(M P a)\end{array}$ & $\begin{array}{c}\text { Ultimate axial compressive strain } \\
\text { of confined concrete } \varepsilon\end{array}$ \\
\hline ACI -17 & $\begin{array}{c}f_{l}=\frac{2 E_{f} n t_{f} \varepsilon_{f e}}{D} \text { if } \frac{f_{l}}{f_{c}^{\prime}} \geq 0.08 \\
\varepsilon_{f e}=k_{e} \varepsilon_{f u} ; k_{e}=0.55\end{array}$ & $\begin{array}{c}f_{c c}^{\prime}=f_{c}^{\prime}+\psi_{f} 3.3 k_{a} f_{l} \\
\qquad k_{a}=1 \\
\text { Stress - Strain Curve: } \\
f_{c c}=\left\{\begin{array}{cc}E_{c} \varepsilon_{c c}-\frac{\left(E_{c}-E_{2}\right)^{2}}{4 f_{c o}^{\prime}} \varepsilon_{c c}^{2} & 0 \leq \varepsilon_{c c} \leq \varepsilon_{t} \\
f_{c}^{\prime}+E_{2} \varepsilon_{c c} & \varepsilon_{t} \leq \varepsilon_{c c} \leq \varepsilon_{c c u}\end{array}\right\} \\
E_{2}=\frac{f_{c c}^{\prime}-f_{c o}^{\prime}}{\varepsilon_{c c u}} ; \varepsilon_{t}=\frac{2 f_{c o}^{\prime}}{E_{c}-E_{2}}\end{array}$ & $\begin{array}{l}\epsilon_{c c u}=\epsilon_{c o}^{\prime}\left(1.50+12 k_{b} \frac{f_{l}}{f_{c}^{\prime}}\left(\frac{\varepsilon_{f e}}{\varepsilon_{c}^{\prime}}\right)^{0.45}\right) \\
k_{b}=1 \\
\epsilon_{c c u} \leq 0.01\end{array}$ \\
\hline AFGC -11 & $\begin{array}{c}f_{l}=E_{p} \varepsilon_{f u d} \\
f_{f u d}=\frac{\alpha_{f} f_{f u}}{\gamma_{f}} \rightarrow \varepsilon_{f u d}=\min \left(\frac{f_{f u d}}{E_{f}} ; 0.85 \%\right) \\
E_{p}=\frac{2 n t_{f}}{D} E_{f}\end{array}$ & $\begin{array}{l}f_{c c}^{\prime}=f_{c}^{\prime}+\psi_{s} \alpha k_{s} k_{w} f_{l} \\
\psi_{s}=0.80 \quad k_{s}=1 \\
\alpha=3.45 \text { for } f_{c}^{\prime} \leq 60 \mathrm{MPa} \\
k_{w}=1 \text { for continuos wrapping }\end{array}$ & Not provided \\
\hline CNR - 13 & $\begin{aligned} f_{l} & =\frac{1}{2} k_{e f f} \rho_{f} E_{f} \varepsilon_{f e} \text { if } \frac{f_{l}}{f_{c}^{\prime}}>0.05 \\
k_{e f f} & =k_{s} k_{V} k_{\alpha} \\
k_{V} & =\left(1-\frac{p_{f}^{\prime}}{2 D}\right)^{2} \\
k_{\alpha} & =\frac{1}{1+\left(\tan \alpha_{f}\right)^{2}} \quad k_{s}=1 \\
\varepsilon_{f e} & =\min \left(\eta_{a} \frac{\varepsilon_{f u}}{\gamma_{f}} ; 0.004\right) \\
\rho_{f} & =\frac{4 n t_{f}}{D} \text { for continuos wrapping }\end{aligned}$ & $\begin{array}{c}f_{c c}^{\prime}=f_{c}^{\prime}\left(1+2.6\left(\frac{f_{l}}{f_{c}^{\prime}}\right)^{2 / 3}\right) \\
\text { Stress - Strain Curve similar to ACI Guideline } \\
\text { (Lam and Teng }(41))\end{array}$ & $\begin{array}{l}\text { (for axial and bending load) } \\
\varepsilon_{c c u}=0.0035+0.015 \sqrt{\frac{f_{l}}{f_{c}^{\prime}}}\end{array}$ \\
\hline CS -12 & $\begin{array}{l}\text { There is not a specific calculation } \\
\text { for the confinement pressure }\end{array}$ & $\begin{array}{c}f_{c c}^{\prime}=0.85 f_{c}^{\prime}\left(1+5.25\left(\rho_{K}-0.01\right) \rho_{\varepsilon}\right) \text { if } \rho_{K} \geq 0.01 \\
\rho_{K}=\frac{2 E_{f} t_{f} n}{\left(\frac{0.85 f_{c}^{\prime}}{\varepsilon_{c}^{\prime}}\right) D} ; \rho_{\varepsilon}=\frac{\varepsilon_{f e}}{\varepsilon_{c}^{\prime}} ; \varepsilon_{f e}=0.6 \varepsilon_{f u} \\
\text { Stress - Strain Curve similar to ACI Guideline } \\
\quad \text { (Lam and Teng (41)) }\end{array}$ & $\begin{array}{c}\varepsilon_{c c u}=\varepsilon_{c o}^{\prime}\left(1.75+6.5 \rho_{K}^{0.8} \rho_{\varepsilon}^{1.45}\right) \\
\qquad \varepsilon_{c c u}<0.01 \\
\text { If } \varepsilon_{c c u}>0.01 \text {, the maximum con- } \\
\text { fined stress }\left(f_{c c}^{\prime}\right) \text { should be taken } \\
\text { the corresponding to this strain } \\
\text { limit. }\end{array}$ \\
\hline CSA - 12 & $\begin{array}{c}f_{l}=\frac{2 n t_{f} f_{f e}}{D} \\
f_{f e}=\min \left(0.006 E_{f} ; \phi_{f} f_{f u}\right)\end{array}$ & $\begin{array}{l}f_{c c}^{\prime}=0.85 f_{c}^{\prime}+k_{l} k_{s} f_{l} \\
k_{l}=6.7\left(k_{s} f_{l}\right)^{-0.17} \\
k_{s}=1\end{array}$ & Not provided \\
\hline fib - 19 & $\begin{array}{l}\text { There is not a specific calculation } \\
\text { for the confinement pressure }\end{array}$ & $\begin{array}{c}f_{c c}^{\prime}=f_{c}^{\prime}+3.3 \frac{2 \mathrm{t}_{\mathrm{f}, \mathrm{t}} E_{f} \varepsilon_{f e}}{D} \text { if } \frac{2 \mathrm{t}_{\mathrm{f}, \mathrm{t}} E_{f} \varepsilon_{f e}}{D f_{c}^{\prime}} \geq 0.07 \\
\varepsilon_{f e}=k_{e} \varepsilon_{f u} \\
k_{\varepsilon}=\left\{\begin{array}{cc}0.5 \frac{R}{50}\left(2-\frac{R}{50}\right) & R<50 \mathrm{~mm} \\
0.5 & R \geq 50 \mathrm{~mm}\end{array}\right\} \\
t_{f, t}=\left\{\begin{array}{cc}n t_{f} & n \leq 3 \\
n^{0.85} t_{f} & n \geq 4\end{array}\right\} \\
\text { Stress - Strain Curve similar to ACI Guide- } \\
\text { line (Lam and Teng (41)) }\end{array}$ & $\epsilon_{c c u}=\epsilon_{c o}^{\prime}\left(1.75+12 \frac{2 \mathrm{t}_{\mathrm{fft}} E_{f} \varepsilon_{f e}}{D f_{c}^{\prime}}\left(\frac{\varepsilon_{f e}}{\varepsilon_{c}^{\prime}}\right)^{0.45}\right)$ \\
\hline ISIS - 08 & $\begin{array}{c}f_{l}=\frac{2 n t_{f} f_{f e}}{D} \rightarrow 0.1 \mathrm{f}_{\mathrm{c}}^{\prime} \leq f_{l} \leq 0.33 \mathrm{f}_{\mathrm{c}}^{\prime} \\
f_{f e}=\min \left(0.004 E_{f} ; \phi_{f} f_{f u}\right)\end{array}$ & $f_{c c}^{\prime}=f_{c}^{\prime}+2 f_{l}$ & Not provided \\
\hline $\begin{array}{l}\text { NCHRP } \\
-10\end{array}$ & $\begin{aligned} f_{l}=\phi_{f} \frac{2 E_{f} \varepsilon_{\mathrm{fe}} n t_{f}}{D} & \rightarrow 4.0 \leq f_{l} \leq \frac{f_{c}^{\prime}}{2}\left(\frac{1}{k_{e} \phi}-1\right) \\
\varepsilon_{\mathrm{fe}} & =0.004\end{aligned}$ & $f_{c c}^{\prime}=f_{c}^{\prime}\left(1+\frac{2 f_{l}}{f_{c}^{\prime}}\right)$ & Not provided \\
\hline TEC-07 & $\begin{array}{r}f_{l}=\frac{1}{2} k_{s} \rho_{f} \varepsilon_{f e} E_{f} \\
k_{s}=1 \quad \rho_{f}=\frac{4 n t_{f}}{D} \\
\varepsilon_{f e}=\min \left(0.5 \varepsilon_{f u} ; 0.004\right)\end{array}$ & $f_{c c}^{\prime}=f_{c}^{\prime}\left(1+2.4\left(\frac{f_{l}}{f_{c}^{\prime}}\right)\right) \geq 1.2 f_{c}^{\prime}$ & $\varepsilon_{c c u}=0.002\left(1+15\left(\frac{f_{l}}{f_{c}^{\prime}}\right)^{0.75}\right)$ \\
\hline
\end{tabular}


TABLE 4. Summary of design guideline' equations for non-circular cross sections.

\begin{tabular}{|c|c|c|c|}
\hline Guideline & Effective confinement pressure $f_{l}(\mathrm{MPa})$ & $\begin{array}{l}\text { Confined concrete compressive strength } \\
\text { for purpose of design } f_{c c}^{\prime} \text { (MPa) }\end{array}$ & $\begin{array}{c}\text { Ultimate axial } \\
\text { compressive strain of } \\
\text { confined concrete } \varepsilon \\
\end{array}$ \\
\hline \multirow{4}{*}{ ACI - 17} & \multirow{2}{*}{$f_{l}=\frac{2 E_{f} n t_{f} \varepsilon_{f_{e}}}{D_{\text {equ }}}$ if $\frac{f_{l}}{f_{t}^{\prime}} \geq 0.08$} & $f_{c c}^{\prime \prime}=f_{c}^{\prime}+\psi_{f} 3.3 k_{a} f_{l}$ & 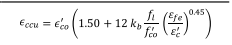 \\
\hline & & $k_{a}=\frac{A_{e}}{A_{c}}\left(\frac{b}{h}\right)^{2}$ & $k_{b}=\frac{A_{e}}{A_{c}}\left(\frac{h}{b}\right)^{0.5}$ \\
\hline & $D_{e q u}=\sqrt{b^{2}+h^{2}}$ & $\left(\left[\frac{b}{h}\right)\left(h-2 r_{c}\right)^{2}+\left(\frac{h}{b}\right)\left(b-2 r_{c}\right)^{2}\right]$ & $\epsilon_{c c u} \leq 0.01$ \\
\hline & $\varepsilon_{f e}=k_{e} \varepsilon_{f u} ; k_{\varepsilon}=0.55$ & $\frac{A_{e}}{\underline{e}}=\frac{\left(1-\frac{1, t i}{3 A_{g}}-\rho_{l}\right)}{1-\rho_{t}}$ & \\
\hline \multirow[b]{2}{*}{$\mathrm{AFGC}-11$} & $f_{l}=E_{p} \varepsilon_{f u d}$ & $f_{c c}^{\prime}=f_{c}^{\prime}+\psi_{s} \alpha k_{s} k_{w} f_{i}$ & \multirow[b]{2}{*}{ Not provided } \\
\hline & $\begin{array}{l}f_{f u d}=\frac{\alpha_{f} f_{f_{u}}}{\gamma_{f}} \rightarrow \varepsilon_{\text {fud }}=\min \left(\frac{f_{\text {rud }}}{E_{f}} ; 0.85 \%\right) \\
E_{p}=\frac{2 n t_{f}}{h} E_{f}\end{array}$ & $\begin{array}{l}\psi_{s}=0.60 \quad \alpha=3.45 \text { for } f_{c}^{\prime} \leq 60 \mathrm{MPa} \\
k_{s}=1-\frac{\left(-2-2 r_{c}\right)^{2}+\left(b-2 b-2 r_{c}\right)^{2}}{3 b h} \\
k_{w}=1 \text { for continuos wrapping }\end{array}$ & \\
\hline \multirow{3}{*}{ CNR -13} & $f_{l}=\frac{1}{2} k_{e f f} \rho_{f} E_{f} \varepsilon_{e} i f \frac{f_{i}}{f_{t}^{\prime}}>0.05$ & & \multirow{3}{*}{$\begin{array}{l}\text { (for axial and bending load) } \\
\varepsilon_{c c u}=0.0035+0.015 \sqrt{\frac{f_{i}^{\prime}}{f_{c}^{\prime}}} \\
\text { The } f_{i} \text { according to: } \\
\varepsilon_{f_{e}}=\eta_{a} \frac{\varepsilon_{f y}}{\gamma_{f}} \leq 0.6 \varepsilon_{f u}\end{array}$} \\
\hline & $\begin{array}{l}k_{e f f}=k_{s} k_{v} k_{\alpha} \\
k_{v}=\left(1-\frac{p_{f}^{\prime}}{2 \cdot \min (b, h)}\right)^{2} \\
k_{\alpha}=\frac{1}{1+\left(\tan \alpha_{f}\right)^{2}} \\
k_{s}=1-\frac{\left(h-2 h r_{c}\right)^{2}+\left(b-2 r_{c}\right)^{2}}{3 b h} \\
\varepsilon_{f e}=\min \left(\frac{\left.\eta_{a} \frac{\varepsilon_{u}}{\gamma_{f}} ; 0.004\right)}{\gamma_{f}}\right)\end{array}$ & $f_{c c}^{\prime}=f_{c}^{\prime}\left(1+2.6\left(\frac{f_{i}^{2}}{f_{c}^{\prime}}\right)^{2 / 3}\right)$ & \\
\hline & $\rho_{f}=\frac{2 n t_{f}(b+h)}{(b h)}$ for continuos wrapping & & \\
\hline \multirow{3}{*}{$\begin{array}{l}\text { CS - 12 } \\
\text { Prismatic }\end{array}$} & \multirow{6}{*}{$\begin{array}{l}\text { There is not a specific calculation for the } \\
\text { confinement pressure. }\end{array}$} & $f_{c c}^{\prime}=0.85 f_{c}^{\prime}\left(1+5.25\left(k_{e} \rho_{K}-0.01\right) \rho_{\varepsilon}\right)$ if $\rho_{K} \geq \frac{0.01}{k_{e}}$ & \multirow{6}{*}{$\epsilon_{c u t} \leq 0.01$} \\
\hline & & $\rho_{K}=\frac{E_{f} t_{t} n}{\left(\frac{0.85}{\varepsilon_{c}^{\prime}}\right)} ; \rho_{c}=\frac{\varepsilon_{e}}{\varepsilon_{c}^{\prime}} ; k_{e}=\frac{r_{c}}{b}\left(1+\frac{b}{h}\right)$ & \\
\hline & & $\varepsilon_{f e}=\varepsilon_{f u}\left[0.46\left(\frac{2 r_{c}}{h}\right)+0.14\right]$ & \\
\hline \multirow{3}{*}{$\begin{array}{l}\text { CS - } 12 \\
\text { Elliptical }\end{array}$} & & $f_{c c}^{\prime}=0.85 f_{c}^{\prime}\left(1+5.25\left(k_{e} \rho_{K}-0.01\right) \rho_{\varepsilon}\right)$ if $\rho_{K} \geq \frac{0.01}{k_{e}}$ & \\
\hline & & $D_{e q v}=\frac{2 a c}{1.5(a+c)-\sqrt{a c}}$ & \\
\hline & & 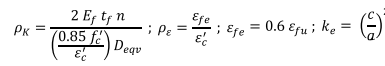 & \\
\hline \multirow{3}{*}{ CSA - 12} & $f_{i}=\frac{2 n t_{t} f_{f e}}{D_{\text {eqv }}}$ & $f_{c c}^{\prime \prime}=0.85 f_{c}^{\prime \prime}+k_{t} k_{s} f_{l}$ & \multirow{3}{*}{ Not provided } \\
\hline & $f_{f e}=\min \left(0.006 E_{f} ; \phi_{f} f_{r}\right)$ & $k_{l}=6.7\left(k_{s} f\right)^{-0.17}$ & \\
\hline & $D_{e q v}=\min (h, b)$ & $k_{s}=0.4$ & \\
\hline fib -19 & $\begin{array}{l}\text { There is not a specific calculation for the } \\
\text { confinement pressure }\end{array}$ & 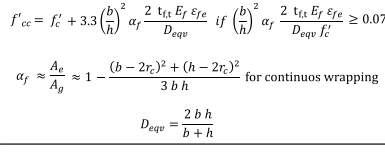 & 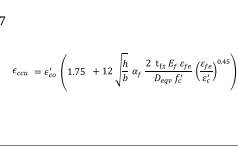 \\
\hline \multirow{4}{*}{ ISIS - 08} & $f_{t}=\frac{2 n t_{f} f_{f e}}{D_{e q u}}$ & & \\
\hline & $D_{e q v}=\sqrt{b^{2}+h^{2}}$ & $f_{c c}^{\prime}=f_{c}^{\prime}+2 f_{i}$ & Not provided \\
\hline & $f_{f e}=\min \left(0.004 E_{f} ; \phi_{f} f_{f u}\right)$ & & \\
\hline & $0.1 \mathrm{f}_{\mathrm{c}}^{\prime} \leq f_{l} \leq 0.33 \mathrm{f}_{\mathrm{c}}^{\prime}$ & & \\
\hline \multirow{3}{*}{ NCHRP - 10} & $f_{l}=\phi_{f} \frac{2 E_{f} \varepsilon_{f e} n t_{f}}{D_{e q v}}$ & & \\
\hline & $\varepsilon_{f e}=0.004$ & $f_{c c}^{\prime}=f_{c}^{\prime}\left(1+\frac{2 f_{i}}{f_{c}^{\prime}}\right)$ & Not provided \\
\hline & $D_{e q v}=\min (h, b)$ & & \\
\hline \multirow{5}{*}{ TEC - 07} & $f_{l}=\frac{1}{2} k_{s} \rho_{f} \varepsilon_{f e} E_{f}$ & \multirow{5}{*}{$f_{c c}^{\prime}=f_{c}^{\prime}\left(1+2.4\left(\frac{f_{l}}{f_{c}^{\prime}}\right)\right) \rightarrow f_{c c}^{\prime} \geq 1.2 f_{c}^{\prime}$} & \multirow{5}{*}{$\varepsilon_{c c u}=0.002\left(1+15\left(\frac{f_{t}}{f_{c}^{\prime}}\right)^{0.75}\right)$} \\
\hline & $\varepsilon_{f e}=\min \left(0.5 \varepsilon_{f u} ; 0.004\right)$ & & \\
\hline & $\begin{array}{ll}\frac{c}{a} & \text { Elliptical } \\
a\end{array}$ & & \\
\hline & $\left\{1-\frac{\left(b-2 r_{c}\right)^{2}+\left(h-2 r_{c}\right)^{2}}{3 b h}\right.$ Rectangular $\}$ & & \\
\hline & $\rho_{f}=\left\{\begin{array}{ll}\left(\frac{2 n t_{f}(b+h)}{(b h)}\right) & \text { Rectangular } \\
\frac{n t_{f}\left(2 \pi \sqrt{\frac{a^{2}+c^{2}}{2}}\right)}{\pi a c} & \text { Elliptical }\end{array}\right\}$ & & \\
\hline
\end{tabular}


- In all the design guidelines except for the AFGC guideline, FRP effective strain is calculated based on FRP ultimate tensile strain (obtained from tensile tests), directly rectifying ultimate strain or via the reduction of FRP ultimate strength. In the AFGC guideline calculations, the ultimate tensile strength is considered directly as it is provided in the guideline. No safety factors are considered when computing actual FRP jacket stress or strain.

- In the parametric study, the confinement expressions are computed as they are provided in the guidelines. However, for the second comparative study, in the first part, both values $f_{c}^{\prime}$ and $0.85 f_{c}^{\prime}$ are considered for comparison reasons. In the second part, unconfined strength is considered as described in each guideline.

- Some guidelines provide maximum and minimum limits for ultimate strain and confinement pressure. In the parametric study, these limits are considered, but in the experimental comparative study, they are not, because a wider range of values needs to be examined and calibrated.

- The parametric study was based on a Carbon-Fibre Reinforced Polymer (CFRP) application. However, its conclusions could be transferable to other types of FRP, taking account of the different elastic modulus ranges of other fibre materials.

- The first part of the experimental comparative study, the column cross-section influence study, is based on a CFRP application. This subsection evaluates how each guideline varies its predictions according to the column cross-section. Therefore, the conclusions can be extrapolated to other types of FRP, considering possible changes with a different elastic modulus.

- The second part of the experimental comparative study is based on an experimental database of medium, large and full-scale concrete column specimens. The majority are strengthened with carbon fibre, followed by glass fibre and finally aramid fibre.

\subsection{Parametric study of the strengthening ratio}

In this subsection, for fully-wrapped columns, the strengthening ratio $\left(f_{c c}^{\prime} / f_{c o}^{\prime}\right)$ provided by each guideline is studied through a parametric study. For circular columns, the studied parameters include column diameter, FRP volumetric ratio $\left(\rho_{f}\right)$ (the relationship between the FRP and concrete cross-sectional areas) and concrete strength. For the rectangular sections, the parametric study focuses on the side-aspect ratio (the relationship between the long and short column sides) and the corner radius. The parametric study is carried out based on a CFRP with the following characteristics: $t_{f}=0.167 \mathrm{~mm}, \varepsilon_{f u}=0.93 \%$ and $E_{f}=291 \mathrm{GPa}$, concrete $=30 \mathrm{MPa}$, in circular sections, diameter $f_{c}^{\prime}=350 \mathrm{~mm}$.

In circular columns, to analyse the influence of diameter, the number of CFRP layers is two (Figure 3 ) and four (Figure 4). For the FRP volumetric ratio influence study (Figure 5), $\rho_{f}$ varies from 0.002 to 0.012 . For the analysis of concrete class influence (Figure 6), $f_{c}^{\prime}$ is between 10 and $60 \mathrm{MPa}$ with $\rho_{f}$ equal to $0.76 \%$. In rectangular columns, the side-aspect ratio influence is analysed in Figure 7. For the study, four CFRP layers were used, with a corner radius of $35 \mathrm{~mm}$ and initial dimensions of $300 \times 300 \mathrm{~mm}$.

\subsubsection{Circular column: influence of diameter}

Column diameter has a direct influence on concrete confinement effectiveness as it changes the FRP

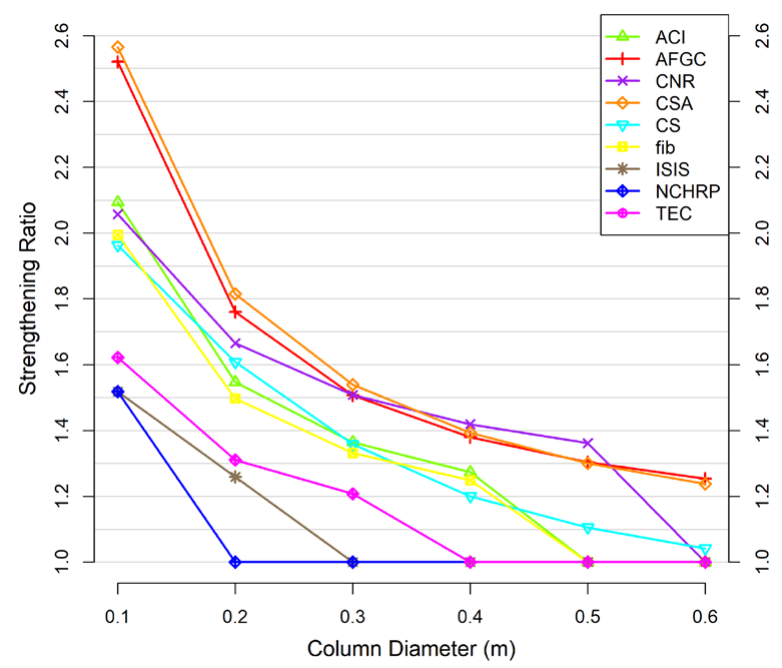

FIGURE 3. Strengthening ratio versus columns diameter for two CFRP layers.

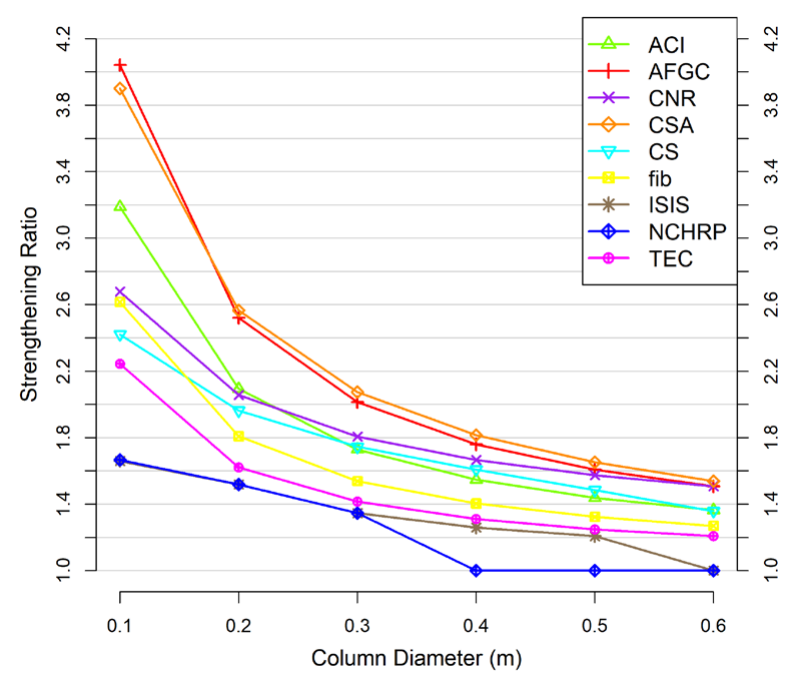

FigURE 4. Strengthening ratio versus columns diameter for four CFRP layers. 
volumetric ratio. The larger the column diameter, the smaller the FRP volumetric ratio, resulting in decreased confined concrete strength (Figures 3 and 4).

As observed in Figures 3 and 4, the prediction curves show a monotonic decreasing trend up to the guideline limit. This limit is linked to a minimum confined lateral FRP pressure, which is required to have sufficient FRP confinement. The ACI, CS, fib, ISIS, NCHRP and TEC guidelines have a minimum limit. Below this limit, there is no increase in confined concrete strength. The NCHRP guideline has the most restrictive minimum limit with FRP confining pressure of $4 \mathrm{MPa}$. In some cases (ACI, CS, ISIS and NCHRP guidelines), an upper limit restricts concrete strength gain. This limit is related to maximum concrete strain, claiming to prevent excessive cracking and the resulting loss of concrete integrity.

With four strengthening layers (Figure 4), there are fewer lower limits than with two strengthening layers (Figure 3). Furthermore, the NCHRP guideline gives the most conservative estimations of the strengthening ratio $\left(f_{c c}^{\prime} / f_{c}^{\prime}\right)$, whilst both the AFGC and CSA guidelines provide the least conservative estimations of $f_{c c}^{\prime} / f_{c}^{\prime}$. The results show significant differences between the guidelines' predictions, with the imposed limits (if they exist) having great significance.

\subsubsection{Circular column: Influence of FRP volumetric ratio and concrete strength}

The curves in Figure 5 correlate the strengthening ratio with the FRP volumetric ratio for the studied guidelines. As observed in Figure 5, the guidelines' curves show an upwards trend. In some cases, their slope changes due to the limits of the guidelines (ACI, CNR, fib, ISIS, NCHRP and TEC). At high FRP volumetric ratios, the AFGC and CSA guidelines are the least conservative as regards the

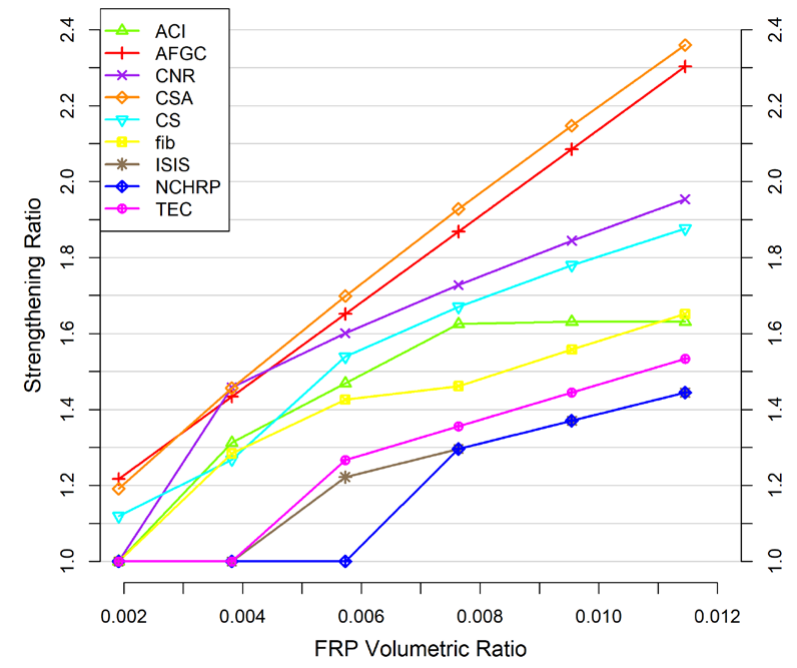

FIGURE 5. Strengthening ratio in circular columns versus FRP volumetric ratio $\left(\rho_{\mathrm{f}}\right)$ for a $30 \mathrm{MPa}$ concrete. strengthening ratio. On the other hand, the ISIS and NCHRP guidelines are the most conservative.

Regarding the influence of concrete strength on the strengthening ratio (Figure 6), all the guidelines show a decreasing strengthening ratio as unconfined concrete strength increases, meaning that the strength enhancement capacity of FRP confinement is higher in concretes with a lower strength class. This statement is in accordance with the literature (22-25). The sharp changes in the guideline curves are due to the design guidelines' limits. Once again, the ISIS and NCHRP guidelines are the most conservative, whereas the CSA and AFGC guidelines have a higher strengthening ratio: 3.6 and 4.1 , respectively for a concrete strength of $10 \mathrm{MPa}$. These two guidelines do not have an upper limit, resulting in a degree of uncertainty. The absence of limits may therefore discourage the use of these guidelines.

\subsubsection{Rectangular column: Influence of the side-as- pect ratio $(h / b)$}

FRP confinement of concrete columns is less effective in non-circular columns than circular columns. Effectiveness in rectangular columns decreases as the side-aspect ratio increases. The design guidelines consider the side-aspect ratio in different ways.

As shown in Figure 7, in all the guidelines, except for the CSA and NCHRP guidelines, the strengthening ratio decreases as the side-aspect ratio increases. The CSA and NCHRP guidelines only use the minimum side dimensions in their calculations. Therefore, confined concrete strength does not change as the aspect ratio increases. The guidelines establish a limit for the aspect ratio. Above this limit, strength enhancement should not be considered. Sharp changes in the guideline trends are due to these aspect ratio limits (AFGC, CSA, CS, ISIS and NCHRP guidelines). The CNR and CSA guidelines

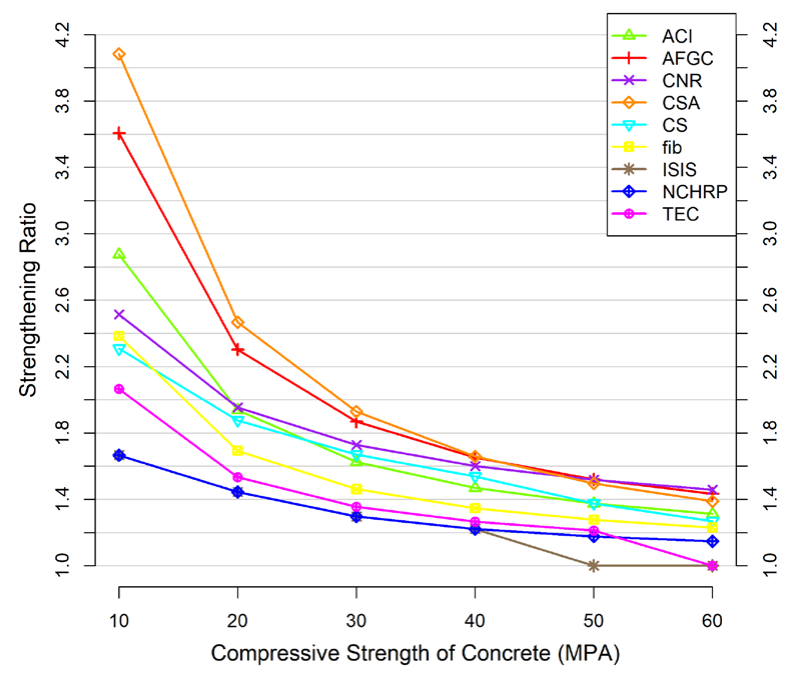

FiguRE 6. Strengthening ratio in circular columns versus unconfined cylinder concrete strength $\left(\mathrm{f}_{\mathrm{c}}^{\prime}\right)$. 


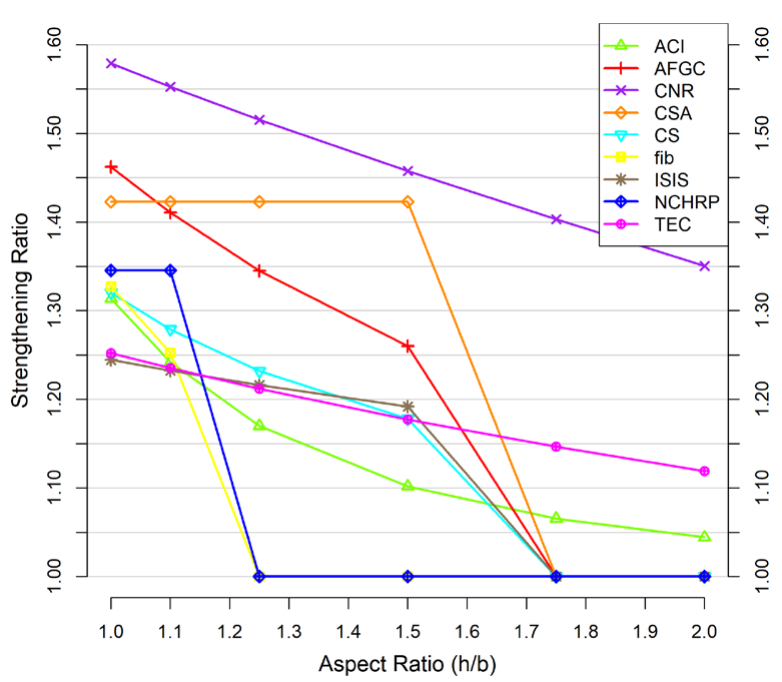

FIGURE 7. Strengthening ratio versus side aspect ratio $(\mathrm{h} / \mathrm{b})$ for rectangular columns.

are the least conservative. The NCHRP guideline is the most restrictive because, due to its side-aspect ratio limit, strength enhancement is only permitted when the aspect ratio is equal to or smaller than 1.1. Both the CSA and NCHRP guidelines have the same strengthening ratio when subject to different aspect ratios since the side-aspect ratio is not a variable in their equations; it is only a limit. The variability of predicted strengthening ratios is less scattered than in previous parametric results for circular cross-section columns.

\subsubsection{Summary of the comparative parametric study}

As per previous results, confined concrete compression strength predictions can be vary greatly between design guidelines. The limits associated with lateral confinement pressure make a great difference to the strength predictions. There are significant variations between the limits of each guideline. There is also no agreement on the non-circular limits for the side-aspect ratio (from 1.1 to 2.0).

The NCHRP and ISIS guidelines give the most conservative strengthening predictions, partly due to their stringent limitation criteria for the side-aspect ratio, corner radius and minimum lateral confinement pressure applied by the FRP, with these limits being $4 \mathrm{MPa}$ and $0.1 f_{c}^{\prime}$, respectively. Overall, the least conservative strengthening ratios are obtained using the AFGC and CSA guidelines. These two guidelines for circular cross-sections provide strengthening ratios much greater than 2 , which is scientifically unsound (58).

To summarise, the guidelines' predictions suggest better strengthening ratios for lower-strength concrete, smaller side-aspect ratios and larger corner radii.

\subsection{Experimental comparative study}

A comparison between the experimental results and the predictive results given by the design guidelines is organised in two stages.

In the first stage, an analysis is conducted on how the guidelines' estimations predict the experimental behaviour of columns with different cross-sections (circular, square and rectangular), where all have an equivalent area and FRP volumetric ratio. This study is based on the experimental results of Rocca et al. (59).

The second stage compares the analysed guidelines with an experimental database of concrete column specimens with different concrete strengths, with the aim of measuring the accuracy of the design guideline predictions for a wider range of concrete characteristic strengths. In this part, unconfined concrete strength is considered as defined by each guideline.

In this experimental comparative study, the upper and lower limits are not considered in the calculations. However, because the confined compressive strength is higher than the unconfined value in all cases, the use of the guidelines' equations is deemed appropriate, even when the minimum lateral confinement pressure limit is not observed.

\subsubsection{Influence of the column cross-section}

In Rocca et al. (59), different guidelines' estimations for circular, square and rectangular cross-section columns, all with an equivalent cross-section area and FRP volumetric ratio, are compared with experimental results. In this paper, the analysed guidelines are extended and some of them are updated as per the latest versions.

To assess the different methods, six column specimens were tested: two circular (C), two square (S) and two rectangular $(\mathrm{R})$ sections. All the cross-sections were quite similar in terms of the cross-sectional area $\left(A_{g}\right)$ and all of them were fully wrapped using the same CFRP composed of two plies, each with thickness of $0.167 \mathrm{~mm}$, ultimate strain of $0.93 \%$ and an elastic modulus of $291 \mathrm{GPa}$. All the rectangular and square specimens had a corner radius of $30 \mathrm{~mm}$. The characteristics of each of the specimens are given in Table 5.

The design guidelines' predictions for the maximum confined axial compressive strength and the ultimate confined axial strain are included in Table 6 . Two sets of results are given, with $f_{c o}^{\prime}=f_{c}^{\prime}$ and $f_{c o}^{\prime}=0.85 f_{c}^{\prime}$. The final column shows whether the calculations meet the limits required by the guidelines. As not all the guidelines have limits or expressions for computing $\varepsilon_{c c u}$, in some cases, NA (Not Applicable) is used.

There are significant differences between the guidelines' predictions for the confined axial compressive strength and the experimental results, as shown in Table 6. For circular cross-sections, if 
TABLE 5. Specimens characteristics, adapted from Rocca et al. (59) and Rocca (61).

\begin{tabular}{|c|c|c|c|c|c|c|c|c|c|c|}
\hline Specimen & $\begin{array}{c}D \\
(\mathrm{~mm})\end{array}$ & $\begin{array}{c}b \\
(\mathrm{~mm})\end{array}$ & $\begin{array}{c}h \\
(\mathrm{~mm})\end{array}$ & $\frac{h}{b}$ & $\begin{array}{c}H \\
(\mathrm{~m})\end{array}$ & $\begin{array}{c}A_{g} \\
\left(\mathrm{~cm}^{2}\right)\end{array}$ & $\begin{array}{c}f_{c}^{\prime} \\
(\mathrm{MPa})\end{array}$ & $\begin{array}{c}\rho_{f} \\
(\%)\end{array}$ & $\begin{array}{l}f_{c o}^{\prime} \\
{\left[f_{c c}^{\prime}\right]}\end{array}$ & $\begin{array}{l}\varepsilon_{c u} \\
{\left[\varepsilon_{c c u}\right]}\end{array}$ \\
\hline $\mathrm{C} 1$ & \multirow{2}{*}{508} & \multirow{2}{*}{-} & \multirow{2}{*}{-} & \multirow{2}{*}{-} & \multirow{2}{*}{1.12} & \multirow{2}{*}{20.3} & 31.7 & 0.00 & 26.3 & 0.003 \\
\hline $\mathrm{C} 2$ & & & & & & & 31.9 & 0.26 & [37.9] & 0.013 \\
\hline $\mathrm{S} 1$ & \multirow{2}{*}{-} & \multirow{2}{*}{458} & \multirow{2}{*}{458} & \multirow{2}{*}{1.0} & \multirow{2}{*}{1.02} & \multirow{2}{*}{20.1} & 32.1 & 0.00 & 26.0 & 0.002 \\
\hline S2 & & & & & & & 32.1 & 0.29 & {$[27.6]$} & 0.003 \\
\hline $\mathrm{R} 1$ & \multirow{2}{*}{-} & \multirow{2}{*}{318} & \multirow{2}{*}{635} & \multirow{2}{*}{2.0} & \multirow{2}{*}{1.37} & \multirow{2}{*}{20.2} & 30.1 & 0.00 & 24.7 & 0.002 \\
\hline $\mathrm{R} 2$ & & & & & & & 30.4 & 0.32 & [24.9] & 0.007 \\
\hline
\end{tabular}

the guideline's predictions are taken as directly expressed, i.e., using $0.85 f_{c}^{\prime}$ as unconfined concrete strength for the CS and CSA guidelines and $f_{c}^{\prime}$ for the others, the ACI and fib guidelines are the most accurate. The AFGC, CNR and CSA guidelines overestimate the confined strength by $7.4 \%, 12.6 \%$ and $6.4 \%$, respectively. The other guidelines $(\mathrm{CS}$, ISIS, NCHRP and TEC) underestimate the confined strength. The underprediction of the NCHRP guideline for circular cross-sections was previously observed by Yazdani et al. (60).

For square and rectangular sections (Table 6), there is clear evidence of the issues the guidelines have with non-circular section predictions. All the guidelines, considering both $0.85 f_{c}^{\prime}$ and $f_{c}^{\prime}$ as $f_{c o}^{\prime}$, overestimate $f_{c c}^{\prime}$. For rectangular sections, the maximum value is obtained via the CSA guideline, with an overestimation of $46.3 \%$ and for square sections, it is obtained via the CNR guideline, with an overestimation of $43.2 \%$.

In the prismatic sections with the assumption of $0.85 f_{c}^{\prime}$ being $f_{c o}^{\prime}$, the guidelines' estimations are closer to the experimental results. One factor that could be attributed to this is that most of the guidelines assume that $f_{c}^{\prime}$ is equal to $f_{c o}^{\prime}$. In the prismatic experimental results, the compressive strength of the unconfined cylinder is $32.1 \mathrm{MPa}$ and $30.3 \mathrm{Mpa}$ for the square and rectangular sections, respectively. These values are already higher than the unconfined strength values obtained experimentally from scaled specimens, which are 26.0 MPa and 24.7 MPa, respectively. Therefore, as the equations mainly start from cylinder compressive strength, the estimations tend to overestimate the unconfined strength value. Zeng et al. (37) found that the compressive strength of concrete in a large-scale unconfined concrete column was lower than that of a standard concrete cylinder, with a ratio of 0.94 between them. Regarding this aspect, more large and medium-scale prismatic and circular specimens should be tested to refine the expressions mainly based on the experimental results of plain confined concrete cylinder tests.

The difference between the unconfined compressive strength of a cylinder and the unconfined compressive strength obtained from the scaled columns
TABLE 6. Design guidelines' previsions for and .

\begin{tabular}{|c|c|c|c|c|c|}
\hline \multicolumn{3}{|c|}{$f_{c o}^{\prime}=f_{c}^{\prime}$} & \multicolumn{3}{|c|}{$f_{c o}^{\prime}=0.85 f_{c}^{\prime}$} \\
\hline Guideline & $\begin{array}{c}f_{c c}^{\prime} \\
(\mathrm{MPa})\end{array}$ & $\varepsilon_{c c u}$ & $\begin{array}{c}f_{c c}^{\prime} \\
(\mathrm{MPa})\end{array}$ & $\varepsilon_{c c u}$ & $\begin{array}{l}\text { Guideline } \\
\text { Limits }\end{array}$ \\
\hline \multicolumn{6}{|c|}{ Circular Cross Section } \\
\hline EXP. DATA & 37.9 & 0.013 & 37.9 & 0.013 & - \\
\hline ACI & 38.2 & 0.005 & 33.4 & 0.006 & NOT OK \\
\hline AFGC & 40.7 & NA & 35.9 & NA & NA \\
\hline CNR & 42.3 & 0.007 & 37.3 & 0.007 & NOT OK \\
\hline CSA & 45.1 & NA & 40.3 & NA & NA \\
\hline $\mathrm{CS}$ & 38.3 & 0.006 & 34.2 & 0.007 & $\mathrm{OK}$ \\
\hline $\mathrm{fib}$ & 37.6 & 0.006 & 32.8 & 0.006 & NOT OK \\
\hline ISIS & 34.8 & NA & 30.0 & NA & NOT OK \\
\hline NCHRP & 34.8 & NA & 30.0 & NA & NOT OK \\
\hline TEC & 35.4 & 0.005 & 30.6 & 0.006 & $\mathrm{OK}$ \\
\hline \multicolumn{6}{|c|}{ Square Cross Section } \\
\hline EXP. DATA & 27.6 & 0.003 & 27.6 & 0.003 & - \\
\hline ACI & 34.6 & 0.004 & 29.8 & 0.004 & NOT OK \\
\hline AFGC & 35.8 & NA & 31.0 & NA & NOT OK \\
\hline CNR & 39.5 & 0.006 & 34.3 & 0.006 & NOT OK \\
\hline CSA & 38.9 & NA & 34.1 & NA & $\mathrm{OK}$ \\
\hline $\mathrm{CS}$ & 34.7 & NA & 30.1 & NA & $\mathrm{OK}$ \\
\hline fib & 35.3 & 0.005 & 30.5 & 0.005 & NOT OK \\
\hline ISIS & 34.5 & NA & 29.7 & NA & NOT OK \\
\hline NCHRP & 35.5 & NA & 30.7 & NA & NOT OK \\
\hline TEC & 34.1 & 0.004 & 29.3 & 0.004 & $\mathrm{OK}$ \\
\hline \multicolumn{6}{|c|}{ Rectangular Cross Section } \\
\hline EXP. DATA & 24.9 & 0.007 & 24.9 & 0.007 & - \\
\hline ACI & 30.8 & 0.004 & 26.3 & 0.004 & NOT OK \\
\hline AFGC & 32.1 & NA & 27.6 & NA & NOT OK \\
\hline $\mathrm{CNR}$ & 36.2 & 0.006 & 31.36 & 0.006 & NOT OK \\
\hline CSA & 41.3 & NA & 36.5 & NA & NOT OK \\
\hline $\mathrm{CS}$ & 33.0 & NA & 26.7 & NA & NOT OK \\
\hline $\mathrm{fib}$ & 30.9 & 0.005 & 26.3 & 0.005 & NOT OK \\
\hline ISIS & 32.4 & NA & 27.9 & NA & NOT OK \\
\hline NCHRP & 35.1 & NA & 30.6 & NA & NOT OK \\
\hline TEC & 31.8 & 0.004 & 27.2 & 0.004 & NOT OK \\
\hline
\end{tabular}


could be due to the size effect phenomenon (62). It is widely accepted that the strength of concrete structures generally decreases as structure size increases (63-67).

The strengthening ratios of the guidelines' predictions and experimental results are shown in Figure 8. The ACI and fib guidelines give the closest predictions to the experimental results for circular sections, but for prismatic sections, they overestimate strength enhancement. Most trends show that, for a similar FRP volumetric ratio, the larger the side-aspect ratio, the smaller the strengthening ratio, with circular sections being more efficient than prismatic ones. However, four guidelines (CSA, CS, ISIS and NCHRP) are not in line with the previous statement and they provide larger strengthening ratios for rectangular sections than square ones.

However, even the ISIS and NCHRP guidelines have prismatic strengthening ratios larger than the circular ones. A main reason for this behaviour is that the CSA and NCHRP guidelines compute lateral pressure depending on an equivalent circular diameter cross-section, where diameter is the minimum dimension of the non-circular cross-section, which is smaller for the prismatic than for the circular cross-section in this case. Another reason is that the NCHRP and ISIS guidelines do not have any type of shape coefficient $\left(k_{s}\right)$ that considers the concentration of confinement pressure on the corners and the resulting decrease in the strengthening ratio. The CS guideline gives almost the same strengthening ratio for the square and the rectangle. This is because in the CS guideline, the confinement effectiveness factor $\left(k_{e}\right)$ and strain efficiency factor $\left(k_{\varepsilon}\right)$ depend on the side-aspect ratio and, in this case, they are quite similar for both cross-sections.

Regarding the ultimate axial compressive strain for confined concrete $\left(\varepsilon_{c c u}\right)$, only five of the nine analysed guidelines give an expression for its computation (only four for the prismatic cross-section). For circular sections, the guidelines' predictions fall

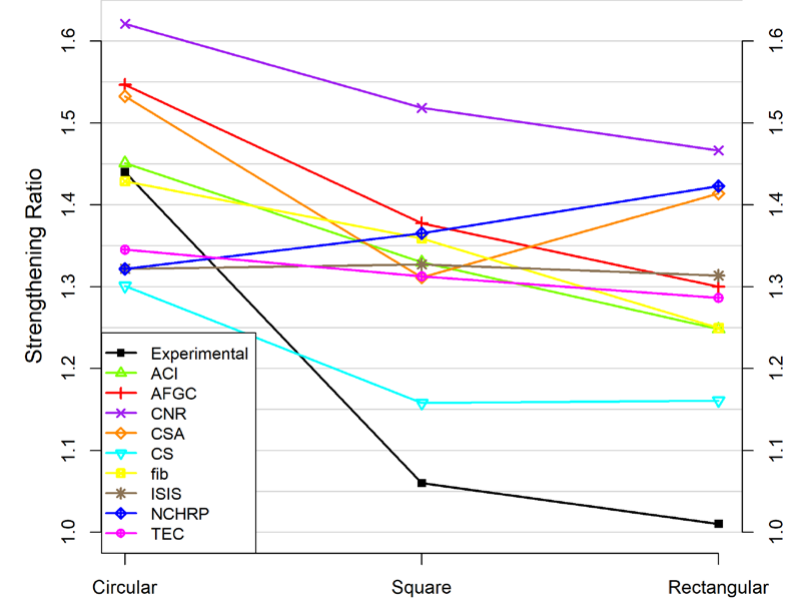

FIGURE 8. Strengthening ratios versus cross-sectional shape. well short of the experimental results, as they are very conservative in their estimations for the $\varepsilon_{c c u}$. Conversely, for the square sections, the predictions overestimate ductility. For the rectangular sections, the predictions are below the experimental results but some of them are closer than for circular sections. The predictions for strain improvement are much more scattered than for strength enhancement, highlighting the difficulty in obtaining ultimate strain due to the localised individualities of the concrete material and FRP jacket.

\subsubsection{Guideline prediction accuracy compared to an experimental database}

The guidelines' equations for FRP strengthening columns are based on confinement models, which are themselves based on experimental tests, generally on small-scale plain concrete specimens. In the second part of the experimental comparative study, to verify the accuracy of the guidelines, their predictions are checked against a database containing the experimental results of a total of 69 specimens, including medium, large and full-scale specimens. Despite the limited number of full-scale experiments, they are critical not only for validating confinement models, but also for generating compelling evidence to support the design guideline methodologies (68).

Experimental results from the previous literature $(37,39,62,68-80)$ are summarised in Tables 7 and 8 for circular and non-circular columns, respectively. All the considered specimens are fully wrapped, with the fibres oriented in the hoop direction only. Different FRP materials are considered: carbon (C), glass (G), aramid (A) and basalt (B). Fabrics with two types of material are classified as hybrid $(\mathrm{H})$. Table 7 , with experimental results for circular columns, includes the following information: diameter (D) and height $(\mathrm{H})$ of specimen, unconfined concrete compressive strength $\left(f_{c}^{\prime}\right)$, longitudinal steel reinforcement ratio $\left(\rho_{l}\right)$, fibre material type, fibre layer thickness $\left(t_{f}\right)$, number of fibre layers $(n)$, FRP elastic modulus $\left(E_{f}\right)$, ultimate or rupture FRP strain $\left(\varepsilon_{f u}\right)$, confined concrete compressive strength $\left(f_{c c}^{\prime}\right)$ and ultimate confined concrete strain $\left(\varepsilon_{c c u}\right)$. Table 8 , for non-circular columns, is similar to Table 7 , but includes the geometric parameters of prismatic sections: short side $(b)$, long side $(h)$ and corner radius $\left(r_{c}\right)$.

Using the aforementioned experimental results from the research and their corresponding guideline predictions, we estimated the ratio between the guideline predictions and the experimental results. Using the results of these ratios, two forest plots are given for circular sections (Figure 9) and non-circular (square and rectangular) sections (Figure 10), respectively. Forest plots provide a graphical summary of the comparison between the guidelines' predictions and the experimental results, the 95\% confidence intervals (CI) of the Mean Ratio (MR) 
TABLE 7. Summary of test results for FRP-wrapped medium, large and full-scale concrete circular columns.

\begin{tabular}{|c|c|c|c|c|c|c|c|c|c|c|c|}
\hline Researcher & $\begin{array}{c}D \\
(\mathrm{~mm})\end{array}$ & $\begin{array}{c}\boldsymbol{H} \\
(\mathrm{mm})\end{array}$ & $\begin{array}{c}f_{c}^{\prime} \\
(\mathbf{M P a})\end{array}$ & $\begin{array}{c}\rho_{l} \\
(\%)\end{array}$ & $\begin{array}{l}\text { Fiber } \\
\text { Type }\end{array}$ & $\begin{array}{c}t_{f} \\
(\mathrm{~mm})\end{array}$ & $\mathbf{n}$ & $\begin{array}{c}E_{f} \\
(\mathbf{G P a})\end{array}$ & $\begin{array}{c}e_{f u} \\
(\%)\end{array}$ & $\begin{array}{c}f_{c c}^{\prime} \\
(\mathbf{M P a})\end{array}$ & $\begin{array}{l}\varepsilon_{c c u} \\
(\%)\end{array}$ \\
\hline \multirow{2}{*}{ Carey and Harries (69) } & 264 & 762 & 38.8 & 1.12 & $\mathrm{C}$ & 1 & 1 & 72.5 & 1.2 & 54.8 & 1.18 \\
\hline & 610 & 1800 & 48.9 & 1.4 & $\mathrm{C}$ & 1 & 3 & 72.5 & 1.2 & 72.9 & 1.04 \\
\hline \multirow{4}{*}{ Demers and Neale (70) } & 300 & 1200 & 25 & 1.4 & $\mathrm{C}$ & 0.3 & 3 & 84 & 1.5 & 36.6 & - \\
\hline & 300 & 1200 & 25 & 3.5 & $\mathrm{C}$ & 0.3 & 3 & 84 & 1.5 & 36.6 & - \\
\hline & 300 & 1200 & 40 & 1.4 & $\mathrm{C}$ & 0.3 & 3 & 84 & 1.5 & 36.6 & - \\
\hline & 300 & 1200 & 40 & 3.5 & $\mathrm{C}$ & 0.3 & 3 & 84 & 1.5 & 36.6 & - \\
\hline Eid et al. (71) & 303 & 1200 & 29.4 & 1.67 & $\mathrm{C}$ & 0.381 & 2 & 78 & 1.34 & 39.2 & - \\
\hline Hadi (72) & 205 & 925 & 57.3 & 2 & $\mathrm{C}$ & 0.533 & 3 & 43.2 & 1.98 & 78.1 & - \\
\hline \multirow{2}{*}{ Kestner (73) } & 508 & 1830 & 32.8 & 1.53 & G & 0.864 & 3 & 23.4 & 1.9 & 38.9 & 0.95 \\
\hline & 508 & 1.830 & 32.8 & 1.53 & $\mathrm{C}$ & 0.165 & 3. & 23.5 & 1.5 & 50 & 1.16 \\
\hline \multirow{5}{*}{ Matthys et al. (74) } & 400 & 2000 & 34.3 & 0.9 & $\mathrm{C}$ & 0.117 & 5 & 198 & 1.19 & 55.3 & 1.11 \\
\hline & 400 & 2000 & 34.3 & 0.9 & $\mathrm{C}$ & 0.235 & 4 & 480 & 0.22 & 54.5 & 0.43 \\
\hline & 400 & 2000 & 34.3 & 0.9 & $\mathrm{G}$ & 0.3 & 6 & 60 & 1.3 & 55.3 & 0.69 \\
\hline & 400 & 2000 & 34.3 & 0.9 & G & 0.3 & 2 & 60 & 1.3 & 37.2 & 0.38 \\
\hline & 400 & 2000 & 34.3 & 0.9 & $\mathrm{H}(\mathrm{G}+\mathrm{C})$ & 0.123 & 4 & 120 & 0.96 & 44.4 & 0.59 \\
\hline \multirow{2}{*}{ Pessiki et al. (75) } & 508 & 1830 & 32.8 & 1.9 & G & - & 3 & $21.6^{*}$ & 1.9 & 38.9 & 0.78 \\
\hline & 508 & 1830 & 32.8 & 1.9 & $\mathrm{C}$ & - & 3 & $38.1 *$ & 1.5 & 50 & 1.13 \\
\hline Rocca et al. (62) & 508 & 1100 & 31.9 & 1.53 & $\mathrm{C}$ & 0.167 & 2 & 291 & 0.93 & 37.9 & 1.23 \\
\hline \multirow{4}{*}{ Thériault et al. (76) } & 304 & 608 & 36 & - & $\mathrm{C}$ & 0.165 & 4 & 230 & 1.5 & 66 & - \\
\hline & 152 & 912 & 36 & - & $\mathrm{C}$ & 0.165 & 2 & 230 & 1.5 & 64 & - \\
\hline & 152 & 902 & 36 & - & G & 1.3 & 3 & 27.6 & 2 & 87 & - \\
\hline & 304 & 1824 & 36 & - & $\mathrm{C}$ & 0.165 & 4 & 230 & 1.5 & 70 & - \\
\hline \multirow{2}{*}{ Wang and Zhang (77) } & 150 & 450 & 47.3 & 1.14 & A & 0.286 & 2 & 118 & 1.77 & 84.3 & 1.62 \\
\hline & 150 & 450 & 51.1 & 1.14 & $\mathrm{~A}$ & 0.286 & 2 & 118 & 1.77 & 88.7 & 1.42 \\
\hline \multirow{2}{*}{ Youseff et al. (78) } & 406 & 813 & 38.4 & 1.5 & $\mathrm{C}$ & 0.584 & 3 & 103.8 & 1.25 & 63.5 & - \\
\hline & 406 & 813 & 47.1 & 1.5 & $\mathrm{C}$ & 0.584 & 3 & 103.8 & 1.25 & 70.6 & - \\
\hline
\end{tabular}

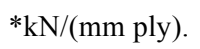

between the guideline's predictions and the experimental results for circular (Figure 9) and non-circular (Figure 10) columns, established based on all analysed scenarios. A more detailed comparison between the guideline prediction and experimental result ratio is given in a panel of boxplots in Figure 11, with the top panel for circular sections and the bottom panel for rectangular sections. A boxplot shows the distribution of data with a central box limited by the $25^{\text {th }}$ and $75^{\text {th }}$ percentiles, which includes a median line reference inside it and has whiskers that correspond to the maximum and minimum values, unless outliers are present, which are shown as discrete points.

Focusing on the circular column, via the MR, we can see that all guidelines underestimate the experimental results. Only the ACI and CNR guidelines included a MR of 1 in the $95 \%$ CI. They can therefore be considered to have an MR equal to 1. The AFGC,
CS, CSA and fib guidelines included an MR of below 1 but over 0.9 , with similar performance. Displaying very different behaviour, the ISIS, NCHRP and TEC guidelines had an MR of under 0.85 in the $95 \% \mathrm{CI}$, clearly failing the hypothesised mean of 1 . To reinforce this analysis, in Figure 11 (upper panel) most boxes of the boxplots can be seen to be below 1 .

The rectangle analysis shows variability in the mean results. The ACI, AFGC, CNR, CSA, fib and NCHRP guidelines overestimate the experimental results. By contrast, the ISIS and TEC guidelines underestimate them. The ISIS and TEC guidelines have a $95 \%$ CI MR value including 1, both with some MR values below 1. The AFGC, CSA and NCHRP guidelines' $\mathrm{CI}$ is above 1 . Therefore, the MR must be considered to be above 1, although the $95 \%$ CI is below 1.2. With a more extreme performance, the CNR, CS and fib guidelines show a 95\% CI of over 1.1 with MR values near to 1.2 . Finally, the ACI 
TABLE 8. Summary of test results for FRP-Wrapped medium, large and full-scale concrete non-circular columns.

\begin{tabular}{|c|c|c|c|c|c|c|c|c|c|c|c|c|c|}
\hline Researcher & $\begin{array}{c}\mathbf{b} \\
(\mathrm{mm})\end{array}$ & $\begin{array}{c}\mathbf{h} \\
(\mathbf{m m})\end{array}$ & $\begin{array}{c}\mathbf{H} \\
(\mathbf{m m})\end{array}$ & $\begin{array}{c}\mathbf{r}_{\mathbf{c}} \\
(\mathbf{m m})\end{array}$ & $\begin{array}{c}\boldsymbol{f}_{c}^{\prime} \\
(\mathbf{M P a})\end{array}$ & $\begin{array}{c}\rho_{l} \\
(\%)\end{array}$ & $\begin{array}{l}\text { Fiber } \\
\text { Type }\end{array}$ & $\begin{array}{c}t_{f} \\
(\mathrm{~mm})\end{array}$ & $\mathbf{n}$ & $\begin{array}{c}E_{f} \\
(G P a)\end{array}$ & $\begin{array}{l}e_{f u} \\
(\%)\end{array}$ & $\begin{array}{c}f_{c c}^{\prime} \\
(\mathrm{MPa})\end{array}$ & $\begin{array}{l}\varepsilon_{c c u} \\
(\%)\end{array}$ \\
\hline \multirow{10}{*}{$\begin{array}{l}\text { Diego et } \\
\text { al. (39) }\end{array}$} & 150 & 150 & 600 & 25 & 8.8 & 0.5 & $\mathrm{C}$ & 0.333 & 1 & 242 & 1.5 & 22.2 & - \\
\hline & 150 & 150 & 600 & 25 & 13 & 0.5 & $\mathrm{C}$ & 0.333 & 1 & 242 & 1.5 & 23.5 & 4.13 \\
\hline & 150 & 150 & 600 & 25 & 16.3 & 0.5 & $\mathrm{C}$ & 0.333 & 1 & 242 & 1.5 & 28.3 & 3.12 \\
\hline & 150 & 150 & 600 & 25 & 16.5 & 0.5 & $\mathrm{C}$ & 0.333 & 1 & 242 & 1.5 & 29.1 & 2.42 \\
\hline & 150 & 150 & 600 & 25 & 17.5 & 0.5 & $\mathrm{C}$ & 0.333 & 1 & 242 & 1.5 & 25.8 & 2.89 \\
\hline & 150 & 150 & 600 & 25 & 8.8 & 0.5 & $\mathrm{G}$ & 0.4 & 2 & 70 & 3.7 & 19.4 & 2.87 \\
\hline & 150 & 150 & 600 & 25 & 13 & 0.5 & $\mathrm{G}$ & 0.4 & 2 & 70 & 3.7 & 19.2 & 3.51 \\
\hline & 150 & 150 & 600 & 25 & 16.3 & 0.5 & G & 0.4 & 2 & 70 & 3.7 & 24.9 & 1.97 \\
\hline & 150 & 150 & 600 & 25 & 16.5 & 0.5 & $\mathrm{G}$ & 0.4 & 2 & 70 & 3.7 & 24.2 & 2.37 \\
\hline & 150 & 150 & 600 & 25 & 17.5 & 0.5 & $\mathrm{G}$ & 0.4 & 2 & 70 & 3.7 & 24.6 & 2.86 \\
\hline \multirow{2}{*}{$\begin{array}{c}\text { Kestner } \\
(73)\end{array}$} & 457 & 457 & 1830 & 38 & 31.5 & 1.48 & $\mathrm{G}$ & 0.864 & 3 & 23.4 & 1.9 & 35.5 & 0.35 \\
\hline & 457 & 457 & 1830 & 38 & 31.5 & 1.48 & $\mathrm{C}$ & 0.165 & & 234.5 & 1.5 & 37.4 & 0.23 \\
\hline \multirow{8}{*}{$\begin{array}{l}\text { Luca et al. } \\
\qquad(68)\end{array}$} & 610 & 610 & 3050 & 20 & 48.6 & 1 & G & 0.246 & 5 & 76.9 & 4.7 & 47.8 & - \\
\hline & 610 & 610 & 3050 & 20 & 37.1 & 1 & G & 0.589 & 2 & 72.4 & 4.5 & 34.9 & - \\
\hline & 610 & 610 & 3050 & 20 & 44.4 & 1 & $\mathrm{H}(\mathrm{G}+\mathrm{B})$ & 0.2 & 8 & 82.9 & 3.9 & 41.9 & - \\
\hline & 508 & 737 & 3050 & 20 & 34.7 & 1 & $\mathrm{H}(\mathrm{G}+\mathrm{B})$ & 0.2 & 8 & 82.9 & 3.9 & 45.4 & - \\
\hline & 356 & 508 & 3050 & 20 & 53.8 & 1 & $\mathrm{G}$ & 0.246 & 5 & 76.9 & 4.7 & 47.9 & - \\
\hline & 356 & 508 & 3050 & 20 & 46.4 & 1 & $\mathrm{G}$ & 0.589 & 2 & 72.4 & 4.5 & 39.3 & - \\
\hline & 356 & 508 & 3050 & 20 & 49.7 & 1 & $\mathrm{G}$ & 0.589 & 5 & 72.4 & 4.5 & 48.3 & - \\
\hline & 356 & 508 & 3050 & 20 & 46.8 & 1 & $\mathrm{H}(\mathrm{G}+\mathrm{B})$ & 0.2 & 8 & 82.9 & 3.9 & 44.1 & - \\
\hline \multirow{10}{*}{$\begin{array}{c}\text { Rocca et } \\
\text { al. (62) }\end{array}$} & 313 & 635 & 1400 & 30 & 30.4 & 1.56 & $\mathrm{C}$ & 0.167 & 7 & 291 & 0.93 & 30.3 & 1.53 \\
\hline & 313 & 635 & 1400 & 30 & 30.4 & 1.56 & $\mathrm{C}$ & 0.167 & 2 & 291 & 0.93 & 24.7 & 0.54 \\
\hline & 457 & 457 & 1000 & 30 & 32.3 & 1.48 & $\mathrm{C}$ & 0.167 & 4 & 291 & 0.93 & 29.1 & 1.1 \\
\hline & 457 & 457 & 1000 & 30 & 32.1 & 1.48 & $\mathrm{C}$ & 0.167 & 2 & 291 & 0.93 & 27.6 & 0.85 \\
\hline & 648 & 648 & 1400 & 30 & 30.9 & 1.48 & $\mathrm{C}$ & 0.167 & 5 & 291 & 0.93 & 30.5 & 0.51 \\
\hline & 648 & 648 & 1400 & 30 & 30.7 & 1.48 & $\mathrm{C}$ & 0.167 & 2 & 291 & 0.93 & 27.2 & 0.42 \\
\hline & 324 & 324 & 700 & 30 & 33 & 1.53 & $\mathrm{C}$ & 0.167 & 2 & 291 & 0.93 & 31.5 & 0.31 \\
\hline & 324 & 324 & 1400 & 30 & 31.5 & 1.53 & $\mathrm{C}$ & 0.167 & 2 & 291 & 0.93 & 30.2 & 0.95 \\
\hline & 914 & 914 & 2000 & 30 & 31.6 & 1.5 & $\mathrm{C}$ & 0.167 & 8 & 291 & 0.93 & 27.5 & 0.81 \\
\hline & 635 & 1270 & 2700 & 30 & 30.3 & 1.52 & $\mathrm{C}$ & 0.167 & 19 & 291 & 0.93 & 28.7 & 0.54 \\
\hline \multirow{3}{*}{$\begin{array}{c}\text { Toutanji et } \\
\text { al. (79) }\end{array}$} & 355 & 355 & 2000 & 30 & 39.1 & 1 & $\mathrm{G}$ & 0.3 & 2 & 60 & 1.3 & 43.8 & 0.37 \\
\hline & 355 & 355 & 2000 & 15 & 37.7 & 1 & $\mathrm{G}$ & 0.3 & 2 & 60 & 1.3 & 41.3 & 0.42 \\
\hline & 355 & 355 & 2000 & 30 & 37.7 & 1 & G & 0.3 & 2 & 60 & 1.3 & 40.6 & - \\
\hline \multirow{2}{*}{$\begin{array}{l}\text { Wang and } \\
\text { Wu (80) }\end{array}$} & 150 & 150 & 450 & 15 & 34.6 & 0 & $\mathrm{~A}$ & 0.286 & 2 & 118 & 1.77 & 54.3 & 0.54 \\
\hline & 150 & 150 & 450 & 15 & 34.6 & 0 & $\mathrm{~A}$ & 0.286 & 2 & 118 & 1.77 & 80.7 & 0.51 \\
\hline \multirow{8}{*}{$\begin{array}{l}\text { Zeng et al. } \\
\text { (37) }\end{array}$} & 290 & 435 & 1300 & 25 & 43.4 & 2.5 & $\mathrm{C}$ & 0.334 & 1 & 245.6 & 1.71 & 46.5 & 0.94 \\
\hline & 290 & 435 & 1300 & 25 & 37.4 & 2.5 & $\mathrm{C}$ & 0.334 & 2 & 245.6 & 1.71 & 42.1 & 1.43 \\
\hline & 290 & 435 & 1300 & 45 & 34.1 & 1.52 & $\mathrm{C}$ & 0.334 & 2 & 245.6 & 1.71 & 42.2 & 2.16 \\
\hline & 290 & 435 & 1300 & 65 & 34.1 & 1.54 & $\mathrm{C}$ & 0.334 & 2 & 245.6 & 1.71 & 44.9 & 2.3 \\
\hline & 290 & 435 & 1300 & 45 & 30.8 & 1.52 & $\mathrm{C}$ & 0.334 & 4 & 245.6 & 1.71 & 45.2 & 2.48 \\
\hline & 290 & 435 & 1300 & 65 & 30.8 & 1.54 & $\mathrm{C}$ & 0.334 & 4 & 245.6 & 1.71 & 51.1 & 2.62 \\
\hline & 290 & 435 & 1300 & 45 & 34.1 & 1.52 & $\mathrm{C}$ & 0.334 & 6 & 245.6 & 1.71 & 63.9 & 3.87 \\
\hline & 290 & 435 & 1300 & 65 & 34.1 & 1.54 & $\mathrm{C}$ & 0.334 & 6 & 245.6 & 1.71 & 68.4 & 4.37 \\
\hline
\end{tabular}




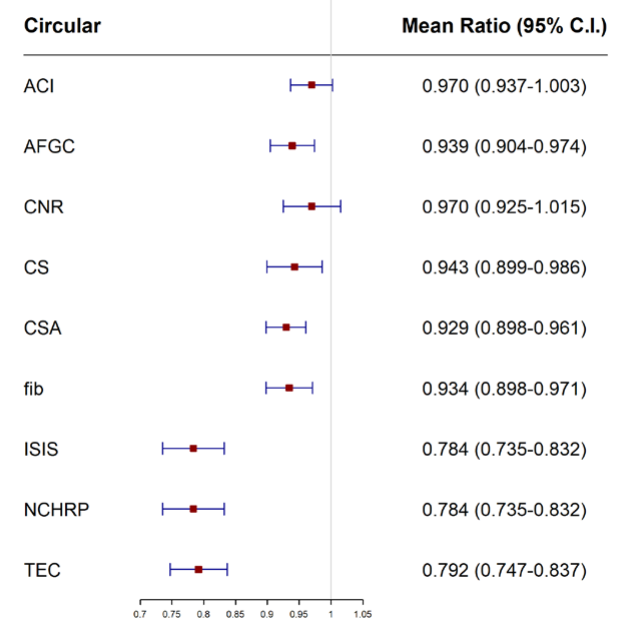

FIGURE 9. Forest plot for circular columns.

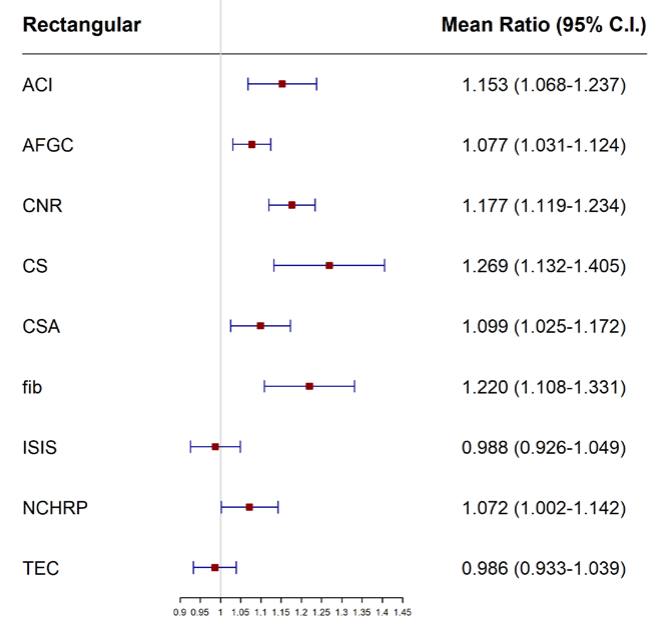

FigURE 10. Forest plot for non-circular columns.

guideline's $95 \% \mathrm{CI}$ is in an intermediate scenario, with an MR value of 1.15. The higher variability in results for non-circular sections is verified in Figure 11 (bottom panel), with most boxes of the boxplots slightly above 1 .

Therefore, it can be stated that for non-circular sections, the guideline estimations are quantitatively worse than for circular predictions. Moreover, for circular columns, the confidence intervals were close to or below 1, whereas for the rectangular estimations, they were above 1 , meaning that the guidelines' predictions overestimate concrete column strengthening $\left(f_{c c, \text { guideline }}^{\prime}>f_{c c, \exp }^{\prime}\right)$.

It seems that the non-circular equations derived from the circular confinement models require more adjustments to give accurate results, as was previously reported by Silva et al. (81). Guler and Ashour (82) and Chaallal et al.(58) also stated that the reliability of the guidelines' predictions varies significantly and that some of them overestimate confined
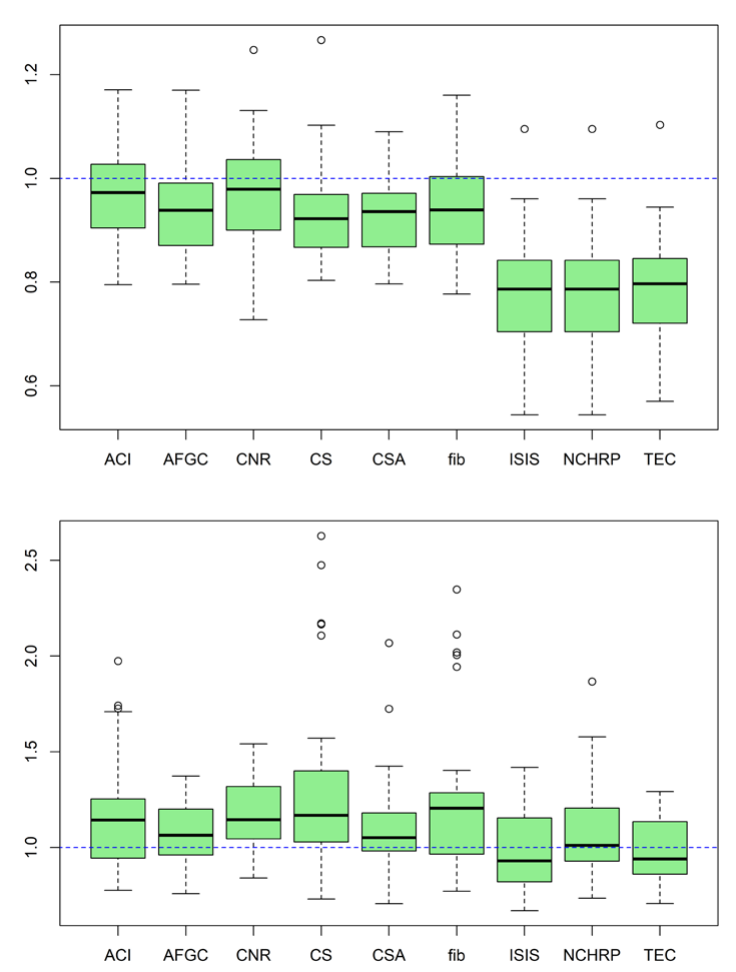

FIGURE 11. Boxplots for circular (top) and non-circular columns (bottom).

concrete strength, which is in line with the results obtained.

Some of the accuracy issues found with the guidelines' predictions can be attributed to the size effect, since most of the guidelines are based on research of standardised cylinder $(150 \times 300 \mathrm{~mm})$ specimens. As such, Carey and Haries (69) determined that the scale of the columns does not significantly affect confinement behaviour for circular columns. Matthys (74) confirmed this idea, stating that although the available models were developed based on small-size cylinders, some of them seem to predict the ultimate strength of large-scale circular columns fairly accurately. Guo et al. (83) stated that the effect of column size is negligible for circular columns. Similar information is provided by Rocca et al. (62), who assert that the size effect within circular cross-section specimens does not appear to be significant but point out the possible influence on non-circular sections. Jin et al. (84) declared that the size effect on the nominal strength of square columns is more significant than on circular columns due to the difference in the constraint effect. Luca et al. (68) also found convergence issues for prismatic concrete columns, which they attributed to dimensional differences between the control concrete cylinder and the as-built unconfined columns.

Other possible causes for the differences between the experimental results and the guidelines' estimations might be that in the design guidelines, the con- 
tribution of steel hoops is not considered. According to Janwaen et al. (31), most of the models only consider the confinement contribution of external FRP wrapping but not the contribution of steel ties. Fanaradelli et al. $(40,85)$ also stated that the differences between the predictions and the experimental results can be attributed to a lack of consideration of internal steel reinforcement and bar buckling for most models. They also stated that future research is needed, including additional parameters and investigating the effects of FRP and steel confinement, as well as the contribution of steel bars and their interactions throughout loading to further improve the predictions.

Another reason for the differences amongst the guidelines is due to the computation of FRP effective strain $\left(\varepsilon_{f e}\right)$. Some of the guidelines limit this value to 0.004 or 0.006 , whilst others apply an efficiency factor $\left(k_{\varepsilon}\right)$. Both of these approaches give more or less similar outcomes for sections reinforced with CFRP. However, for Glass-Fibre Reinforced Plastic (GFRP) the results differ significantly. Glass fibre has higher ultimate strain compared to carbon fibre and, applying a maximum effective strain value, the results are well below the outcomes obtained by applying the efficiency factor. Martínez et al. (33) pointed out that the FRP hoop ultimate strain was much lower than the material's ultimate tensile strain obtained from flat coupon tests and the strain efficiency factor obtained in the tests was less than the value usually recommended by the design guidelines.

To summarise, it can be stated that for circular columns it appears that the stress-strain behaviour of FRP-confined concrete is now well understood and can be accurately predicted, but the situation is different for square and rectangular columns (86). For non-circular sections, the size effect seems to matter and further experimental research needs to be conducted on large-scale columns, as this research is very limited to date $(63,84,87,88)$.

\section{CONCLUSIONS}

This paper provides an analytical review of the design guidelines for FRP-confined concrete columns. Considering that the research conducted is mainly based on CFRP strengthening applications, the following conclusions can be made:

- External FRP confinement can significantly increase the strength of concrete columns subject to axial compressive stress.

- The predictions of the ACI, AFGC, CNR, CS, CSA and fib guidelines for the axial compressive strength of confined concrete are generally suitable for circular columns.

- The guidelines' predictions on concrete confinement compressive strength for non-circular col- umns are inaccurate. There is great disparity in the guidelines' predictions.

- The scattering of predictions for strain improvement (ductility) is larger than for strength enhancement.

- The size effect on the nominal strength of square columns is more significant than on circular columns. To better calibrate the models and design guidelines, more experimental results from large-scale non-circular specimens are required.

- Most of the analysed guidelines show a better strengthening ratio for circular columns than for non-circular ones, in line with the literature. Nonetheless, the CSA, NCHRP and ISIS guidelines do not follow this trend, which contradicts the literature and the experimental evidence.

\section{ACKNOWLEDGEMENTS}

The authors are grateful for the support of the Spanish Government (MCIN), Project Ref. PID2020-119015GB-C22.

The authors would also like to thank the Engineering School of La Almunia (University of Zaragoza) and the AMADE research group (University of Girona).

The authors would like to thank the anonymous reviewers for critically reviewing the manuscript and making substantial improvements.

\section{NOTATION}

The following symbols are used in this paper:

$A_{c}=$ cross-sectional area of concrete in compression member

$A_{e}^{c}=$ cross-sectional area of effectively confined concrete

$A^{e}=$ gross area of concrete section

$A^{g}=$ longitudinal steel reinforcement area

$C_{E}=$ Environmental reduction factor (ACI)

$D=$ diameter of circular cross section

$D_{\text {eq }}=$ equivalent diameter for non-circular cross section

$E_{2}^{e q v}=$ slope of linear portion of confined concrete stress-strain curve

$E=$ initial modulus of elasticity of concrete

$E_{f}^{c}=$ tensile modulus of elasticity of FRP

$E_{p}^{f}=$ FRP confinement stiffness (AFGC)

$L^{p}=$ unsupported length of column

$a=$ major cross sectional dimension on elliptical column

$b=$ short side dimension of rectangular cross section or minor cross sectional dimension on elliptical column

$f=$ confinement pressure due to FRP jacket

$f_{c}=$ characteristic compressive strength of concrete, obtained from standard cylinder

$f^{\prime}{ }_{c c}=$ compressive strength of confined concrete

$f^{,}, c c=$ compressive strength of confined concrete
$f^{c o}=$ compressive strength of unconfined concrete

$f^{, c o}=$ compressive strength of confined concrete at ultimate strain $f_{f e} \stackrel{c u}{=}$ effective tensile strength of FRP, stress attained at section failure

$f_{f u}=$ ultimate tensile strength of FRP, obtained from flat coupon. $f_{f u}=$ ultimate tensile strength of FRP material as reported by the manufacturer (ACI)

$k_{1}=$ confinement coefficient

$k_{2}^{1}=$ strain coefficient 
$k_{H}=$ coefficient of horizontal efficiency $(\mathrm{CNR})$

$k_{V}=$ coefficient of vertical efficiency (CNR)

$k^{V}=$ efficiency factor for FRP reinforcement in determination of $\mathrm{f}^{g}$ (AcI)

$k_{b} \stackrel{\mathrm{cc}}{=}$ efficiency factor for FRP reinforcement in determination of $\varepsilon_{\text {ccu }}$ (ACI)

$k_{e}^{\text {ccu }}=$ Strength reduction factor applied for unexpected eccentricities, equal to 0.85 (spiral columns) or 0.8 (tied columns (NCHRP). For Concrete Society is a confinement effectiveness factor

$k_{\text {eff: }}$ coefficient efficiency (CNR)

$k_{l}^{\text {eff }}=$ confinement effectiveness parameter, $=6.7\left(\mathrm{k} \mathrm{f}^{-0.17}(\mathrm{CSA})\right.$

$k_{\mathrm{s}}=$ shape factor, accounting for the geometry of cross section

$k_{w}^{\mathrm{s}}=$ partial wrapping confinement factor (AFGC)

$k^{w}=$ coefficient of efficiency related to the angle of fibers (CNR)

$k=$ strain efficiency factor for FRP

$\mathrm{h}^{\varepsilon}=$ long side dimension of rectangular cross section

$\mathrm{n}=$ number of FRP plies composing the reinforcement

$p^{\prime}=$ spacing of FRP sheets in a partial wrapping $(\mathrm{CNR})$

$\mathrm{r}_{\mathrm{c}}=$ corner radius of prismatic cross sections

$t_{f}^{c}=$ nominal thickness of one ply of FRP reinforcement

$\alpha=$ confinement efficiency factor (AFGC)

$\alpha_{f}=$ angle of FRP fiber respect to perpendicular of column axis

(CNR), ageing factor (AFGC) and a confinement effectiveness factor in non-circular sections (fib).

$\gamma=$ partial safety factor for concrete (c), FRP (f) and steel (s)

$\varepsilon_{c}=$ strain in concrete

$\varepsilon^{c}=$ compressive strain of unconfined concrete corresponding to $\mathrm{f}^{\prime}$

$\varepsilon_{c c u} \stackrel{\mathrm{c}}{=}$ ultimate axial compressive strain of confined concrete corresponding

$\varepsilon_{f e}=$ FRP effective strain (strain level reached at failure)
$\varepsilon_{f u}=$ ultimate tensile strain of the FRP obtained from flat coupon.

$\varepsilon_{t}=$ transition strain in stress-strain curve of FRP confined con-

crete

$\eta_{\alpha}=$ environmental factor $(\mathrm{CNR})$

$\rho_{f=}$ FRP volumetric ratio, relation between the FRP and concrete cross sectional areas

$\rho_{K}=$ confinement stiffness ratio (Concrete Society)

$\rho_{l}=$ ratio longitudinal steel reinforcement area to cross-sectional

area of a compression member $=\mathrm{A} / \mathrm{A}$

$\rho_{\varepsilon}=$ confinement strain ratio (Concrete Society)

$\varphi=$ strength reduction factor (ACI)

$\varphi=$ resistance factor for concrete (c), FRP (f) and steel (s)

$\Psi_{f}=$ additional FRP strength reduction factor (ACI)

\section{AUTHOR CONTRIBUTIONS:}

Conceptualization: A. Salesa, L.M. Esteban, C. Barris. Data Curation: A. Salesa, L.M. Esteban. Formal Analysis: A. Salesa, L.M. Esteban, C. Barris. Funding acquisition: C. Barris. Investigation: A. Salesa, L.M. Esteban, C. Barris. Methodology: A. Salesa, L.M. Esteban, C. Barris. Project Administration: A. Salesa. Software: L.M. Esteban. Supervision: C. Barris. Validation. C. Barris. Visualization: L.M. Esteban. Roles/Writing, original draft: A. Salesa. Writing, review \& editing: A. Salesa, L.M. Esteban, C. Barris.

\section{REFERENCES}

1. Calavera Ruiz, J. (2005) Patología de estructuras de hormigón armado y pretensado. INTEMAC, Instituto Técnico de Materiales y Construcciones.

2. Fernández-Cánovas, M.; González-García, M.N.; Piñero, J.Á.; Cobo, A. (2016) Compressive strength behaviour of low- and medium-strength concrete specimens confined with carbon fibres in defective implementation conditions: an experimental study. Mater. Construcc. 66 [324], e103. https:// doi.org/10.3989/mc.2016.08315.

3. Tarabia, A.M.; Albakry, H.F. (2014) Strengthening of RC columns by steel angles and strips. Alex. Eng. J. 53 [3], 615626. https://doi.org/10.1016/j.aej.2014.04.005.
4. Maaddawy, T.E. (2009) Strengthening of eccentrically loaded reinforced concrete columns with fiber-reinforced polymer wrapping system: experimental investigation and analytical modeling. J. Compos. Constr. 13 [1], 13-24. https://doi. org/10.1061/(ASCE)1090-0268(2009)13:1(13).

5. Aire, C.; Gettu, R.; Casas, J. R.; Marques, S.; Marques, D (2010) Concrete laterally confined with fibre-reinforced polymers (FRP): experimental study and theoretical model. Mater. Construcc. 60 [297], 19-31. https://doi.org/10.3989/ mc.2010.45608.

6. Fam, A.; Qie, F.S.; Rizkalla, S. (2004) Concrete-filled steel tubes subjected to axial compression and lateral cyclic loads. J. Struct. Eng. 130 [4], 631-640. https://doi.org/10.1061/ (ASCE)0733-9445(2004)130:4(631).

7. Motavalli, M.; Czaderski, C. (2007) FRP Composites for retrofitting of existing civil structures in Europe: State-ofthe-art review. Presented at the Composites \& Polycon 2007, Tampa, FL USA: American Composites Manufacturers Association.

8. Nanni, A. (2003) North American design guidelines for concrete reinforcement and strengthening using FRP: principles, applications and unresolved issues. Constr. Build. Mater. 17 [6-7], 439-446. https://doi.org/10.1016/S0950$0618(03) 00042-4$

9. Kurrer, K.E. (2018) The history of the theory of structures Searching for equilibrium. John Wiley \& Sons.

10. Considère, A. (1902) Résistance à la compression du béton armé et du béton fretté. Le Génie Civil, XLII(1064,1065,1066,1067,1 068,1069,1072), 5-7,20-24,38-40,58-60,72-74,82-86,140.

11. Considère, A. (1902) Étude théorique de la résistance à la compression du béton fretté. Le Ciment: son emploi et ses applications nouvelles, (7e Anné-No9), 133-136.

12. Considère, A. (1902) Etude expérimentale de la résistance à la compression du béton fretté. Le Ciment: son emploi et ses applications nouvelles, (7e Anné-No10), 150-153.

13. Richart, F.E.; Brandtzæg, A.; Brown, R.L. (1928) A study of the failure of concrete under combined compressive stresses. University of Illinois Engineering Experiment Station, Urbana - Champaign, IL. Bulletin 185.

14. Mander, J.B.; Priestley, M.J.N.; Park, R. (1988) Theoretical stress-strain model for confined concrete. J. Struct. Eng. 114(8), 1804-1826. https://doi.org/10.1061/(ASCE)07339445(1988)114:8(1804).

15. Suhail, R.; Amato, G.; McCrum, D.P. (2020) Active and passive confinement of shape modified low strength concrete columns using SMA and FRP systems. Compos. Struct. 251, 112649. https://doi.org/10.1016/j. compstruct.2020.112649.

16. Teng, J.G.; Jiang, T. (2008) 6 - Strengthening of reinforced concrete (RC) columns with fibre-reinforced polymer (FRP) composites. In L. C. Hollaway \& J. G. Teng (Eds.), Strengthening and rehabilitation of civil infrastructures using fibre-reinforced polymer (FRP) Composites (pp. 158-194). Woodhead Publishing. https://doi. org/10.1533/9781845694890.158.

17. Lam, L.; Teng, J.G. (2004) Ultimate condition of fiber reinforced polymer-confined concrete. J. Compos. Constr. 8 [6], 539-548. https://doi.org/10.1061/(ASCE)10900268(2004)8:6(539).

18. Lam, L.; Teng, J.G. (2003) Design-oriented stressstrain model for FRP-confined concrete. Constr. Build Mater. 17 [6-7], 471-489. https://doi.org/10.1016/S0950$0618(03) 00045-X$

19. International Federation for Structural Concrete (fib). (2001) fib 14 Externally Bonded FRP Reinforcement for RC Structures: Technical report on the design and use of externally bonded fibre reinforced polymer reinforcement (FRP EBR) for reinforced concrete structures (Bulletin No. 14). Switzerland: fib.

20. Realfonzo, R.; Napoli, A. (2011) Concrete confined by FRP systems: Confinement efficiency and design strength models. Compos. Part. B-Eng. 42 [4], 736-755. https://doi. org/10.1016/j.compositesb.2011.01.028

21. Ozbakkaloglu, T.; Lim, J.C.; Vincent, T. (2013) FRP-confined concrete in circular sections: Review and assessment of stress-strain models. Eng. Struct. 49, 1068-1088. https://doi. org/10.1016/j.engstruct.2012.06.010. 
22. Gora, A.M.; Jaganathan, J.; Amwar, M.P.; Leung, H.Y. (2018) Experimental studies and theoretical models for concrete columns confined with FRP composites: a review. World. J. Eng. https://doi.org/10.1108/WJE-01-2018-0026.

23. Almusallam, T.H. (2007) Behavior of normal and highstrength concrete cylinders confined with E-glass/epoxy composite laminates. Compos. Part. B-Eng. 38 [5], 629-639. https://doi.org/10.1016/j.compositesb.2006.06.021.

24. Ozbakkaloglu, T.; Oehlers, D.J. (2008) Concrete-filled square and rectangular FRP tubes under axial compression. J. Compos. Constr. 12 [4], 469-477. https://doi.org/10.1061/ (ASCE)1090-0268(2008)12:4(469).

25. Wang, L-M.; Wu, Y-F. (2008) Effect of corner radius on the performance of CFRP-confined square concrete columns: Test. Eng. Struct. 30 [2], 493-505. https://doi.org/10.1016/j. engstruct.2007.04.016.

26. Teng, J.G.; Huang, Y.L.; Lam, L.; Ye, L.P. (2007) Theoretical model for fiber-reinforced polymer-confined concrete. $J$. Compos. Constr. 11 [2], 201-210. https://doi.org/10.1061/ (ASCE)1090-0268(2007)11:2(201).

27. Jiang, T.; Teng, J.G. (2007) Analysis-oriented stress-strain models for FRP-confined concrete. Eng. Struct. 29 [11], 2968-2986. https://doi.org/10.1016/j.engstruct.2007.01.010.

28. Tamuzs, V.; Tepfers, R.; Zile, E.; Ladnova, O. (2006) Behavior of concrete cylinders confined by a carbon composite 3. Deformability and the ultimate axial strain. Mech. Compos. Mater. 42, 303-314. https://doi.org/10.1007/ s11029-006-0040-5.

29. de Diego Villalón, A. (2016) Comportamiento de pilares de hormigón armado confinados con materiales compuestos sometidos a compresión centrada (PhD Thesis). E.T.S.I. Caminos, Canales y Puertos (UPM).

30. Lam, L.; Teng, J.G. (2003) Design-oriented stress-strain model for FRP-confined concrete in rectangular columns. J. Reinf. Plast. Compos. 22, 1149-1186. https://doi. org/10.1177/0731684403035429.

31. Janwaen, W.; Barros, J.A.; Costa, I.G. (2019) A new strengthening technique for increasing the load carrying capacity of rectangular reinforced concrete columns subjected to axial compressive loading. Compos. Part. B-Eng. 158, 6781. https://doi.org/10.1016/j.compositesb.2018.09.045

32. Rochette, P.; Labossière, P. (2000) Axial testing of rectangular column models confined with composites. J. Compos. Constr. 4 [3], 129-136. https://doi.org/10.1061/(ASCE)10900268(2000)4:3(129).

33. Martínez, S.; de Diego, A.; Castro, V.J.; Echevarría, L.; Barroso, F.J.; Rentero, G.; Soldado, R.P.; Gutiérrez, J.P. (2020) Strengthening of low-strength concrete columns with fibre reinforced polymers. Full-scale tests. Infrastructures. 5 [11], 91. https://doi.org/10.3390/infrastructures5110091.

34. Mirmira, A; Shahawy, M.; Samaan, M.; Echary, H.E.; Mastrapa, J.C.; Pico, O. (1998) Effect of column parameters on FRP-confined concrete. J. Compos. Constr. 2 [4], 175-185. https://doi.org/10.1061/(ASCE)10900268(1998)2:4(175).

35. Ilki, A.; Peke, O.; Karamuk, E.; Demir, C.; Kumbasar, N. (2008) FRP retrofit of low and medium strength circular and rectangular reinforced concrete columns. J. Mater. Civ. Eng. 20 [2], 169-188. https://doi.org/10.1061/(ASCE)08991561(2008)20:2(169)

36. Rocca, S.; Galati, N.; Nanni, A. (2006) Large-size reinforced concrete columns strengthened with carbon FRP: experimental evaluation. In Proceedings of the Third International CICE Conference (pp. 491-494). Presented at the Third International Conference on FRP Composites in Civil Engineering (CICE 2006), Miami, USA: International Institute for FRP in Construction (IIFC).

37. Zeng, J.J.; Lin, G.; Teng, J.G.; Li, L.J. (2018) Behavior of large-scale FRP-confined rectangular RC columns under axial compression. Eng. Struct. 174, 629-645. https://doi. org/10.1016/j.engstruct.2018.07.086

38. Maalej, M.; Tanwongsval, S.; Paramasivam, P. (2003) Modelling of rectangular RC columns strengthened with FRP. Cem. Concr. Compos. 25 [2], 263-276. https://doi. org/10.1016/S0958-9465(02)00017-3

39. de Diego, A.; Arteaga, A.; Fernández, J.; Perera, R.; Cisneros, D. (2015) Behaviour of FRP confined concrete in square columns. Mater. Construcc. 65 [320], e069. https://doi. org $/ 10.3989 / \mathrm{mc} .2015 .05414$.

40. Fanaradelli, T.; Rousakis, T.; Karabinis, A. (2019) Reinforced concrete columns of square and rectangular section, confined with FRP - Prediction of stress and strain at failure. Compos. Part. B-Eng. 174, 107046. https://doi.org/10.1016/j. compositesb.2019.107046.

41. ACI Committee 440. (2017) ACI 440.2R-17 Guide for the design and construction of externally bonded FRP systems for strengthening concrete structures. USA: American Concrete Institute.

42. Association Française de Génie Civil. (2011) AFGC Réparation et renforcement des structures en béton au moyen des matériaux composites - Recommandations provisoires. France: AFGC.

43. CNR-Advisory Committee on technical recommendations for construction. (2013) CNR-DT 200 R1/2013 Guide for the design and construction of externally bonded FRP systems for strengthening existing structures. Italy: Italian National Research Council.

44. Concrete Society. (2012) CS-TR55 Design guiadance for strengthening concrete structures using fibre composite materials (Technical Report No. TR55). UK: Concrete Society.

45. Canadian Standards Association. (2012) CSA S806-12 Design and construction of building structures with fibre-reinforced polymers. Canada: Canadian Standards Association.

46. International Federation for Structural Concrete (fib). (2019) Externally applied FRP reinforcement for concrete structures. FIB - International Federation for Structural Concrete.

47. Kenneth, N. (2008) ISIS Design Manual No. 4 FRP Rehabilitation of reinforced concrete structures (Design Manual No. 4). Canada: ISIS Canada.

48. Zureick, A.H.; Ellingwood, B.R.; Nowak, A.S.; Mertz, D. Triantafillou, T.C. (2010) NCHRP-Report 655 Recommended guide specification for the design of externally bonded FRP systems for repair and strengthening of concrete bridge elements (No. 655). USA: Transportation Research Board National Cooperative Highway Research Program.

49. Turkish Ministry of Public Works and Settlement. (2007) TEC-2007 Turkish Earthquake Code (2007): Regulations on structures constructed in disaster regions. Turkey.

50. Légeron, F.; Paultre, P. (2003) Uniaxial confinement model for normal- and high-strength concrete columns. J. Struct. Eng. 129 [2], 241-252. https://doi.org/10.1061/(ASCE)07339445(2003)129:2(241).

51. Ozbakkaloglu, T.; Saatcioglu, M. (2004) Rectangular stress block for high-strength concrete. ACI Struct. J. 101 [4], 475483. https://doi.org/10.14359/13333.

52. ACI Committee 318. (2014) ACI 318-14 Building code requirements for structural concrete. USA: American Concrete Institute.

53. American Association of State Highway and Transportation Officials. (2007) AASHTO LRFD Bridge design specifications (Bulletin No. 4th Edition). Washington, D.C. USA: American Association of State Highway and Transportation Officials.

54. Spoelstra, M.R.; Monti, G. (1999) FRP-confined concrete model. J. Compos. Constr. 3 [3], 143-150. https://doi. org/10.1061/(ASCE)1090-0268(1999)3:3(143).

55. Teng, J.G.; Jiang, T.; Lam, L.; Luo, Y.Z. (2009) Refinement of a design-oriented stress-strain model for FRP-confined concrete. J. Compos. Constr. 13 [4], 274-278. https://doi. org/10.1061/(ASCE)CC.1943-5614.0000012.

56. Teng, J.G.; Lam, L. (2002) Compressive behavior of carbon fiber reinforced polymer-confined concrete in elliptical columns. J. Struct. Eng. 128 [12], 1535-1543. https://doi. org/10.1061/(ASCE)0733-9445(2002)128:12(1535).

57. Triantafillou, T.C.; Choutopoulou, E.; Fotaki, E.; Skorda, M.; Stathopoulou, M.; Karlos, K. (2016) FRP confinement of wall-like reinforced concrete columns. Mater. Struct. 49, 651-664. https://doi.org/10.1617/s11527-015-0526-5.

58. Chaallal, O.; Hassan, M.; LeBlanc, M. (2006) Circular columns confined with FRP: experimental versus predictions of models and guidelines. J. Compos. Constr. 10 [1], 4-12. https://doi.org/10.1061/(ASCE)1090-0268(2006)10:1(4).

59. Rocca, S.; Galati, N.; Nanni, A. (2008) Review of design guidelines for FRP confinement of reinforced concrete 
columns of noncircular cross sections. J. Compos. Constr. 12 [1], 80-92. https://doi.org/10.1061/(ASCE)10900268(2008)12:1(80).

60. Yazdani, N.; Beneberu, E.; Mohiuddin, A.H. (2018) CFRP retrofit of concrete circular columns: Evaluation of design guidelines. Compos. Struct. https://doi.org/10.1016/j. compstruct.2018.02.066.

61. Rocca, S. (2007) Experimental and analytical evaluation of FRP-confined large size reinforced concrete columns. Doctoral Dissertations - University of Missouri-Rolla - USA.

62. Rocca, S.; Galati, N.; Nanni, A. (2008) Experimental evaluation of noncircular reinforced concrete columns strengthened with CFRP. In Seismic strengthening of concrete buildings using FRP composites (pp. 37-56). Michigan, USA: American Concrete Institute.

63. Jin, L.; Chen, H.; Wang, Z.; Du, X. (2020) Size effect on axial compressive failure of CFRP-wrapped square concrete columns: Tests and simulations. Compos. Struct. 254, 112843. https://doi.org/10.1016/j.compstruct.2020.112843.

64. Ince, R.; Arici, E. (2004) Size effect in bearing strength of concrete cubes. Constr. Build. Mater. 18 [8], 603-609. https:// doi.org/10.1016/j.conbuildmat.2004.04.002

65. Carpinteri, A.; Puzzi, S. (2007) Fractal, statistics and size effect in concrete. In Proceedings of the 6th international conference on fracture mechanics of concrete and concrete structures (Vol. 01-03). Presented at the Fracture mechanics of concrete and concrete structures, Catania (Italy): International Association of Fracture Mechanics for Concrete and Concrete Structures.

66. Burtscher, S.; Chiaia, B.; Dempsey, J.P.; Ferro, G.; Gopalaratnam, V.S.; Prat, P.; Rokugo, K.; Saouma, V.E.; Slowik, V.; Vitek, L.; Willam, K. (2004) RILEM TC QFS 'Quasibrittle fracture scaling and size effect'-final report. Mater. Struct. 37, 547-568. https://doi.org/10.1007/ BF02481579.

67. Saouma, V.E.; Fava, G. (2006) On fractals and size effects. Int. J. Fract. 137, 231-249. https://doi.org/10.1007/s10704005-3060-6.

68. de Luca, A.; Nardone, F.; Matta, F.; Nanni, A.; Lignola, G.P.; Prota, A. (2011) Structural evaluation of full-Scale FRPconfined reinforced concrete columns. J. Compos. Constr. 15 [1], 112-123. https://doi.org/10.1061/(ASCE)CC.19435614.0000152

69. Carey, S.A.; Harries, K.A. (2005)Axial behavior and modeling of confined small-, medium-, and large-scale circular sections with carbon fiber-reinforced polymer jackets. ACI Struct. J. 102 [4], 596-604. https://doi.org/10.14359/14564.

70. Demers, M.; Neale, K.W. (1999) Confinement of reinforced concrete columns with fibre-reinforced composite sheets an experimental study. Can. J. Civ. Eng. 26 [2], 226-241. https://doi.org/10.1139/198-067.

71. Eid, R.; Roy, N.; Paultre, P. (2009) Normal- and high-strength concrete circular elements wrapped with FRP composites. $J$. Compos. Constr. 13 [2], 113-124. https://doi.org/10.1061/ (ASCE)1090-0268(2009)13:2(113).

72. Hadi, M.N.S. (2006) Comparative study of eccentrically loaded FRP wrapped columns. Compos. Struct. 74 [2], 127135. https://doi.org/10.1016/j.compstruct.2005.03.013.

73. Kestner, J. (1998) Rehabilitation of reinforced concrete columns using fiber reinforced polymer composite jackets. Theses and dissertations of Lehigh preserve institutional repository.

74. Matthys, S.; Toutanji, H.; Audenaert, K.; Taerwe, L. (2005) Axial load behavior of large-scale columns confined with fiber-reinforced polymer composites. ACI Struct. J. 102 [2], 258-267. https://doi.org/10.14359/14277.

75. Pessik, S.; Harries, K.A.; Kestner, J.T.; Sause, R.; Ricles, J.M. (2001) Axial behavior of reinforced concrete columns confined with FRP jackets. J. Compos. Constr. 5 [4], 237-245. https://doi.org/10.1061/(ASCE)1090-0268(2001)5:4(237).

76. Thériault, M.; Neale, K.W.; Claude, S. (2004) Fiberreinforced polymer-confined circular concrete columns: investigation of size and slenderness effects. J. Compos. Constr. 8 [4], 323-331. https://doi.org/10.1061/(ASCE)10900268(2004)8:4(323)

77. Wang, Y.; Zhang, D. (2009) Creep-effect on mechanical behavior of concrete confined by FRP under axial compression. J. Eng. Mech. 135 [11], 1315-1322. https://doi. org/10.1061/(ASCE)0733-9399(2009)135:11(1315).

78. Youssef, M.N.; Feng, M.Q.; Mosallam, A.S. (2007) Stressstrain model for concrete confined by FRP composites. Compos. Part. B-Eng. 38 [5-6], 614-628. https://doi. org/10.1016/j.compositesb.2006.07.020

79. Toutanji, H.; Han, M.; Gilbert, J.; Matthys, S. (2010) Behavior of large-scale rectangular columns confined with FRP composites. J. Compos. Constr. 14 [1], 62-71. https:// doi.org/10.1061/(ASCE)CC.1943-5614.0000051.

80. Wang, Y-f.; Wu, H-l. (2011) Size effect of concrete short columns confined with aramid FRP jackets. J. Compos. Constr. 15 [4], 535-544. https://doi.org/10.1061/(ASCE) CC.1943-5614.0000178.

81. Lobo, P.S.; Faustino, P.; Jesus, M.; Marreiros, R. (2018). Design model of concrete for circular columns confined with AFRP. Compos. Struct. 200, 69-78. https://doi.org/10.1016/j. compstruct.2018.05.094.

82. Guler, S.; Ashour, A. (2016) Review of current design guidelines for circular FRP-wrapped plain concrete cylinders. J. Compos. Constr. 20 [2], 04015057. https://doi.org/10.1061/ (ASCE)CC.1943-5614.0000619.

83. Guo, Y-C.; Gao, W-Y.; Zeng, J-J.; Duan, Z-J.; Ni, X-Y.; Peng, K-D. (2019) Compressive behavior of FRP ringconfined concrete in circular columns: Effects of specimen size and a new design-oriented stress-strain model. Constr. Build. Mater. 201, 350-368. https://doi.org/10.1016/j. conbuildmat.2018.12.183

84. Jin, L.; Li, X.; Fan, L.; Du, X. (2020) Size effect on compressive strength of GFRP-confined concrete columns: numerical simulation. J. Compos. Constr. 24 [5], 04020038. https://doi.org/10.1061/(ASCE)CC.1943-5614.0001041.

85. Fanaradelli, T.; Rousakis, T. (2020) Assessment of analytical stress and strain at peak and at ultimate conditions for fiber-reinforcement polymer-confined reinforced concrete columns of rectangular sections under axial cyclic loading. Struct. Concr. 22 [1], 95-108. https://doi.org/10.1002/ suco. 201900386

86. Lin, G.; Teng, J.G. (2020) Advanced stress-strain model for FRP-confined concrete in square columns. Compos. Part. B-Eng. 197, 108149. https://doi.org/10.1016/j. compositesb.2020.108149.

87. de Diego, A.; Arteaga, Á.; Fernández, J. (2019) Strengthening of square concrete columns with composite materials. Investigation on the FRP jacket ultimate strain. Compos. Part B-Eng. 162, 454-460. https://doi.org/10.1016/j. compositesb.2019.01.017.

88. Kaeseberg, S.; Messerer, D.; Holschemacher, K. (2019) Assessment of standards and codes dedicated to CFRP confinement of RC columns. Materials. 12 [15], 2390. https://doi.org/10.3390/ma12152390. 